\title{
Room Temperature Synthesis and Antibacterial Activity of New Sulfonamides Containing N,N-Diethyl-Substituted Amido Moieties
}

\author{
Olayinka O. Ajani, ${ }^{1}$ Oluwole B. Familoni, ${ }^{2}$ Feipeng Wu, ${ }^{3}$ \\ Johnbull O. Echeme, ${ }^{1}$ and Zheng Sujiang ${ }^{4}$ \\ ${ }^{1}$ Department of Chemistry, Covenant University, Canaanland, P.M.B. 1023, Ogun State, Ota, Nigeria \\ ${ }^{2}$ Department of Chemistry, University of Lagos, Lagos State, Akoka 100001, Nigeria \\ ${ }^{3}$ New Functional Polymeric Material Group, Technical Institute of Physics and Chemistry, Chinese Academy of Sciences (CAS), \\ Beijing 100190, China \\ ${ }^{4}$ Test Center of Antimicrobial Materials, Technical Institute of Physics and Chemistry, Chinese Academy of Sciences (CAS), \\ Beijing 100190, China
}

Correspondence should be addressed to Olayinka O. Ajani, ola.ajani@covenantuniversity.edu.ng

Received 26 July 2012; Accepted 5 September 2012

Academic Editor: Patrick Bednarski

Copyright ( $\odot 2012$ Olayinka O. Ajani et al. This is an open access article distributed under the Creative Commons Attribution License, which permits unrestricted use, distribution, and reproduction in any medium, provided the original work is properly cited.

\begin{abstract}
Sulfonamide drugs which have brought about an antibiotic revolution in medicine are associated with a wide range of biological activities. We have synthesized a series of $\alpha$-tolylsulfonamide, 1-11 and their substituted $N, N$-diethyl-2(phenylmethylsulfonamido) alkanamide derivatives, 12-22 in improved and excellent yields in aqueous medium at room temperature through highly economical synthetic routes. The chemical structures of the synthesized compounds 1-22 were confirmed by analytical and spectral data such as IR, ${ }^{1} \mathrm{H}$ - and ${ }^{13} \mathrm{C}-\mathrm{NMR}$, and mass spectra. The in vitro antibacterial activity of these compounds along with standard clinical reference, streptomycin, was investigated on two key targeted organisms. It was observed that 1-(benzylsulfonyl)pyrrolidine-2-carboxylic acid, 2 emerged as the most active compound against Staphylococcus aureus at MIC value of $1.8 \mu \mathrm{g} / \mathrm{mL}$ while 4-(3-(diethylamino)-3-oxo-2-(phenylmethylsulfonamido) propyl)phenyl phenylmethanesulfonate, 22 was the most active sulfonamide scaffold on Escherichia coli at MIC value of $12.5 \mu \mathrm{g} / \mathrm{mL}$.
\end{abstract}

\section{Introduction}

The development of sulfonamides is a fascinating and informative area in medicinal chemistry [1-3]. Its functional group has a long and rich history in organic chemistry and drug discovery $[4,5]$. The $p$-toluenesulfonamide and benzenesulfonamides have been widely explored in synthetic chemistry $[4,5]$; however, few work has been done on the $\alpha$ tolylsulfonamide. For instance, synthesis of benzenesulfonamide derivative of pipecolic acid [6] and that of glycine [7] had been reported. In addition, benzenesulfonamide of alanine was achieved as a result of synthetic usage of such amino acid as a linker to $6 H-1,3,4$-thiadiazine scaffold [8] while naphthylsulfonamide was prepared as antagonist of chemokine receptor [9]. Domagk's discovery of antibacterial activity for the azo dye prontosil led to the first effective chemotherapeutic agent, sulfanilamide [10]. A retrospective look at sulfonamides leaves no doubt that besides providing the first effective treatment of bacterial infections $[10,11]$, they also unleashed an antibiotic revolution in medicine [12-15] to rationally design new therapeutic agents [16, 17]. These compounds provided an excellent lead for structural modification and ushered in the modern era of chemotherapy and drug design. Sulfonamides inhibit the multiplication of bacteria by acting as competitive inhibitors of $p$-aminobenzoic acid (PABA) in the folic acid metabolism cycle $[18,19]$. In fact, the discovery that sulfonamides act through folate inhibition resulted in the development of dihydrofolate reductase inhibitors such as trimethoprim $[20$, 21]. 
Furthermore, sulfonamide moiety has a crucial functionality because of its wide variety of reported biological [22-25] and pharmacological activities such as anticancer [26-28], carbonic anhydrase inhibitory [29-31], antibacterial [32$34]$, antimalarial [35, 36], antitumor [37, 38], antihypertensive [39], anti-inflammatory [40-42], and antiprotozoal activities [43]. Some sulfonamides have been established as potent drugs in treatment of insomnia and other sleepless challenges in man by antagonizing orexin neural activity [44-46]. Sulfonamidophenyl porphyrins have a great potential utility as model for activatable photosensitizers [47]. Sulfonamide has also been reported to possess good herbicidal [48] and corrosion inhibitory properties $[49,50]$. Multidrug resistance is one of the major immediate threats to human health today [51]. For instance, methicillin is a good antibacterial agent, yet, methicillin resistance among Staphylococcus aureus [52] and Staphylococcus epidermidis $[53,54]$ as well as other drug resistance in Escherichia coli [55] had been identified to be of great concern in public health. Epidemiological studies have also revealed that the emergent of new diseases is on the increase and quite alarming [56, 57].

In addition, the choice of $S$. aureus and E. coli as the targeted organisms in this study was due to wide range of infectious diseases and life threatening conditions associated with such isolates. S. aureus, which produces heat stable toxin, is among the invasive gram positive known as pyogenic cocci implicated in several diseases of human [58, 59]. From the literatures, $S$. aureus had shown to be very resistant to a wide variety of antibiotics [60]. Infections caused by methicillin-resistant S. aureus (MRSA) and vancomycinresistant $S$. aureus are associated with high morbidity and mortality, high treatment cost, and long stays in hospitals [60]. E. coli, a facultative anaerobe of wide distribution in the environment, has been implicated in the cause of urinary tract infections, meningitis, sepsis, wound infections, nosocomial pneumonia, and arthritis. A subgroup enterohemorrhagic E. coli (EHEC) can cause severe potentially fatal illness known as hemorrhagic colitis with symptoms of blood diarrhea and severe abdominal pain [61].

Based on the numerous applications of sulfonamides and challenges associated with drug usage and multidrug resistance microorganisms aforementioned among others, there is a continuous need for the synthesis of new organic compounds as potential antimicrobial agents for the replacement of the old existing ones currently available in the market or to enhance the potency of the former ones. Thus, it is conceivable to develop a series of functionalized sulfonamides in order to investigate the in vitro antibacterial activities of such scaffolds on the targeted organisms, namely, S. aureus and E. coli.

\section{Results and Discussion}

2.1. Chemistry. In the continuation of our effort on the discovery of rationally designed therapeutic agents [62, 63], we have herein reported the synthesis of a series of new alkylarylsulfonamide with potent antibacterial activity.
We started with the reaction of equimolar proportion of readily available amino acid, L-pipecolic acid with $\alpha$ toluene sulfonylchloride $(\alpha$-TsCl) via a continuous magnetic stirring at room temperature in the presence of aqueous sodium carbonate for $48 \mathrm{~h}$ according to a known procedure [64]. After the completion of the reaction which was TLC monitored, the excess unreacted $\alpha$-TsCl was recovered with dichloromethane (DCM) using separatory funnel while the aqueous layer was worked up by acidifying to a $\mathrm{pH}$ of 2.2 to get clear solution which upon freeze drying and column purification afforded 1-(benzylsulfonyl)piperidine2 -carboxylic acid 1 in excellent yield (98\%). Being motivated by this encouraging discovery, we then proceeded on the coupling of nine other amino acids with $\alpha$-Ts Cl under similar condition to obtain $\alpha$-tolylsulfonamides $\mathbf{2 - 1 0}$ in good to excellent yields (Scheme 1).

In contrast to monosulfonylation that occurred in formation of 1-10, the synthesis of $\mathbf{1 1}$ was achieved in higher yield only when disulfonylation of the required amino acid (tyrosine) was utilized. This was noticed when monosulfonylation of tyrosine afforded $\mathbf{1 1}$ in lower yield (45\%) when compared with other sulfonamide products 110 with their yields ranging from $87.5 \%$ to $98.8 \%$. This was because under the monosulfonylation of tyrosine, the reaction terminated when the $\alpha$-TsCl, being the limiting reagent, has been exhausted living excess of the tyrosine in the aqueous medium. This was as a result of two nucleophilic attacking sites available on the L-tyrosine moiety. This means that the phenolate anion was competing with the amino group in terms of the nucleophilic potential on the $\alpha$ $\mathrm{TsCl}$ precursor, thereby resulting in an unusually lower yield $(45 \%)$. Based on this development, the stoichiometric ratio was revisited in the production of $\mathbf{1 1}$ to give allowance for selective disulfonylation of tyrosine. Hence, the reaction for the production of $\mathbf{1 1}$ was reconducted in such a way that the molar equivalent of $\alpha$-TsCl was doubled that of tyrosine in order to accommodate nucleophilicity from both $\mathrm{NH}_{2}$ and $\mathrm{PhO}^{-}$of the latter, then the yield for $\mathbf{1 1}$ increased drastically to $89.6 \%$ (Scheme 2). Structures of all eleven $\alpha$ tolylsulfonamides were confirmed by spectroscopic means which include IR, mass spectra, ${ }^{1} \mathrm{H}$, and ${ }^{13} \mathrm{C} \mathrm{NMR}$ as well as the elemental analytical data. Thus, the detail structural elucidation was here-in confirmed using compound $\mathbf{1}$ as the representative of the $\alpha$-tolylsulfonamide templates. The infrared spectrum of compound $\mathbf{1}$ gave rise to the absorption bands of $\mathrm{OH}$ and carbonyl of carboxylic acid at 3422 and $1736 \mathrm{~cm}^{-1}$, respectively. Also, the $\mathrm{CH}$ stretching of both aliphatic and aromatic occurred at the vibrational frequency of 2974 and $2822 \mathrm{~cm}^{-1}$, respectively. The two IR bands for $\mathrm{SO}_{2}$ unit were observed at 1159 and $1238 \mathrm{~cm}^{-1}$ as expected, whereas the band accountable for $\mathrm{C}=\mathrm{C}$ of aromatic was observed at $1603 \mathrm{~cm}^{-1}$. The only easily accountable band resulting from a bending vibrational mode at the finger print region was $\mathrm{Ar}-\mathrm{H}$ which appeared at $700 \mathrm{~cm}^{-1}$.

In the ${ }^{1} \mathrm{H}-\mathrm{NMR}$ of compound 1 , all the five aromatic protons resonated downfield as a singlet at $\delta 7.52 \mathrm{ppm}$ while the $\mathrm{CH}_{2}$ of benzylic group appeared as a two-proton singlet at $\delta 4.28 \mathrm{ppm}$. Considering the upfield region, it was discovered that $\mathrm{CH}$ proton adjacent to $\mathrm{COOH}$ resonated 
<smiles>CC(N)C(=O)OCc1ccccc1CS(=O)(=O)N1CCCCC1C(=O)O</smiles>

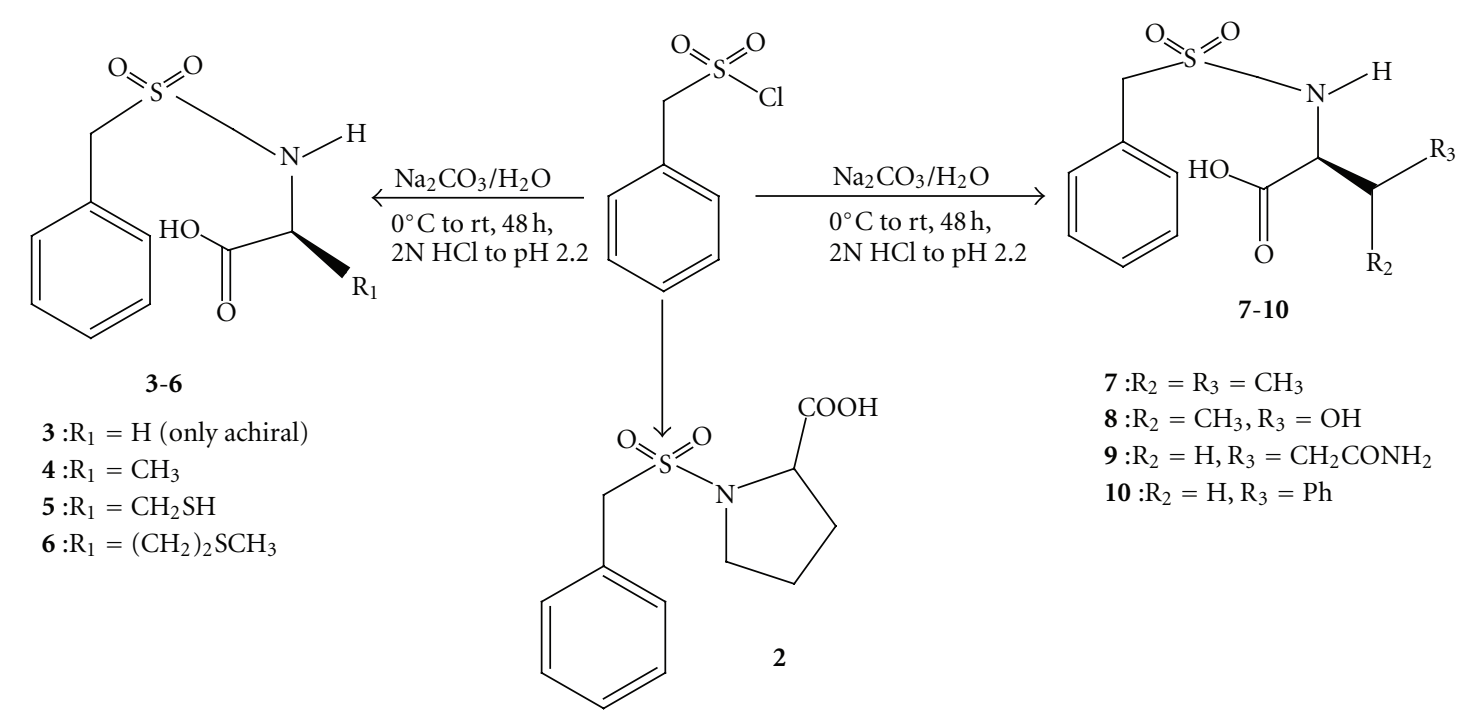

Scheme 1: Synthesis of $\alpha$-tolylsulfonamide derivatives $\mathbf{1 - 1 0 .}$
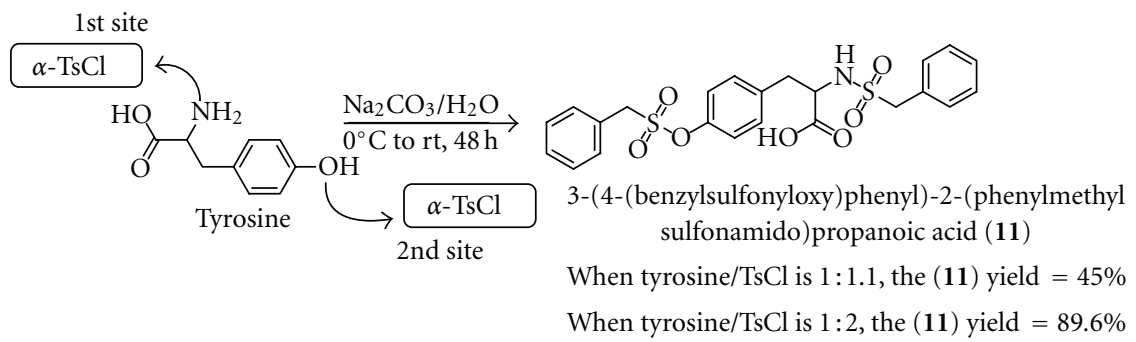

SCHEme 2: Synthesis of sulfonamide 11 by selective disulfonylation of tyrosine.

as a doublet of doublet at $\delta 4.08-4.02 \mathrm{ppm}$ with coupling constants of $3.44 \mathrm{~Hz}$ and $15.12 \mathrm{~Hz}$. This was as a result of the fact that its $\mathrm{CH}_{2}$ neighbouring protons were not totally chemically equivalent even though they were attached to the same carbon atom. All other remaining pipecolic protons were well accounted for upfield between $\delta 3.57-3.53$ and $1.81-1.68 \mathrm{ppm}$. The justification for their appearance near TMS scale lies in the fact that they were attached to $\mathrm{sp}^{3}$ hybridized centre. Although, the molecular ion peak was not observed, the base peak, however, occurred at $\mathrm{m} / \mathrm{z} 178.1$ which was as a result of the loss of benzylic radical. Other prominent peaks which were formed as a result of some fragmentation patterns appeared at $\mathrm{m} / \mathrm{z} 180.1,179.1,165.1$, $121.0,77.0$, and 64.0 with the intensities of $55 \%, 65 \%, 30 \%$, $42 \%, 13 \%$, and $31.7 \%$, respectively.

The second stage of this experiment involved the chemical transformation of the carboxylic acid side chain moieties of the sulfonamides 1-11 into $\mathrm{N}, \mathrm{N}$-diethyl alkanamides 1222 using highly efficient nonconventional method in its slightly modified version [65]. The reaction optimization for this aspect was carried out using amination of $\mathbf{1}$ with diethyl amine under triethyl amine basified condition as a representative. Thus, having established the $C$-amidoalkylation of the sulfonamide $\mathbf{1}$ to achieve the alkanamide $\mathbf{1 2}$ in higher 
yield as a bountiful success, we were motivated to extend our investigation to the reaction of other $\alpha$-tolylsulfonamides 211 with diethylamine under the same condition (Scheme 3 ). These attempts also afforded the N,N-diethyl-substituted products 13-22 in good to excellent yields as envisaged. The spectroscopic assignment was consistent with that of proposed structures for the obtained $N, N$-diethyl alkanamides 12-22. So, it is necessary to consider the spectral data of $\mathbf{1 2}$ as a typical representative of the $N, N$-diethyl alkanamides in order to authenticate such compounds. The IR spectrum of 12 had no absorption band above $3028 \mathrm{~cm}^{-1}$ indicating the absence of $-\mathrm{OH}$ bond. This in turn confirmed the effective amidation of $\mathrm{COOH}$ of compound 1 . The bands at 3028 and $2951 \mathrm{~cm}^{-1}$ depicted $\mathrm{CH}$ of aromatic and aliphatic, respectively. Also, the $\mathrm{C}=\mathrm{O}$ and $\mathrm{C}=\mathrm{C}$ frequency appeared at 1720 and $1593 \mathrm{~cm}^{-1}$, respectively, while the two bands of $\mathrm{SO}_{2}$ were observed at 1188 and $1148 \mathrm{~cm}^{-1}$. Furthermore, the chemical shifts and multiplicity patterns of ${ }^{1} \mathrm{H}$ - and ${ }^{13} \mathrm{C}$ NMR correlated well with that of the proposed alkylated sulfonamides 12-22. The ${ }^{1} \mathrm{H}-\mathrm{NMR}$ spectrum of 12 in $\mathrm{D}_{2} \mathrm{O}$ showed five aromatic protons as singlet at $\delta 7.54$ and $\mathrm{CH}_{2}$ of benzylic group as singlet at $\delta 4.30$. One triplet at $\delta 1.40-$ $1.37(6 \mathrm{H})$ was assigned to two methyl of diethyl group that were chemically equivalent while one quartet at $\delta 3.21-3.16$ $(4 \mathrm{H})$ was assigned to two methylene protons adjacent to the methyl protons with a coupling constant of $7.20 \mathrm{~Hz}$. The ${ }^{13} \mathrm{C}$ NMR of 12 in $\mathrm{D}_{2} \mathrm{O}$ in the presence of a drop of dioxane revealed the presence of seventeen different carbon atoms with $\mathrm{C}=\mathrm{O}$ having the highest signal at $\delta 173.5 \mathrm{ppm}$ while the $\mathrm{CH}_{3}$ carbon atoms appeared to have the least signals at $\delta 11.4 \mathrm{ppm}$.

2.2. Antibacterial Activity. The general antibacterial sensitivity testing (inhibition zone, $\mathrm{mm}$ ) of all the series of twenty-two synthesized sulfonamides alongside with that of streptomycin clinical standard was assayed on test organisms (E. coli and $S$. aureus) using agar diffusion technique [66]. The choice of $E$. coli as the gram - ve organism is because it is easily transmissible through food, water, soil, animal, and man [60]. E. coli is a normal flora of human body which causes a lot of vancomycin-resistant Enterococci and methicillin-resistant Staphylococcus aureus (MRSA) [51]. Based on our previous report [62], the choice of streptomycin as clinical standards is due to the fact that, at low concentrations, streptomycin only inhibits the growth of the bacteria through induction of prokaryotic ribosomes to misread $m$ RNA [67] and it also possesses broad spectrum of antibacterial activity. There were reported cases of E. coli and Staphylococcus aureus being susceptible to streptomycin [4, 68]. The biological relevance of the synthesized sulfonamides here-in was authenticated by screening them in vitro against Staphylococcus aureus ATCC 6538 (S. aureus) and Escherichia coli ATCC 25922 (E. coli) with the reported selectivity index (SI) duly calculated from zones of inhibition (ZOI). Hence, it should be noted afterwards that the abbreviated forms were given in the bracket for the sake of brevity and conciseness.

From the result of sensitivity testing, probable activities of $\alpha$-tolylsulfonamide family on the test organisms were categorized based on the size of zone of inhibition (Table 1). Interestingly, it was observed that some of the compounds exhibited probable significant activities based on the large zone of inhibition reported. For instance, compounds 6, 7, 12,14 , and 22 were highly active on $E$. coli while compounds $1,2,4,5,10,11,17$, and 20 exhibited moderate activities on the same organism. All other compounds showed low activities on $E$. coli except 3 and 16 which showed no activity at all on the $E$. coli even at $1000 \mu \mathrm{g} / \mathrm{mL}$. The scenario of comparative study of effect of the sulfonamides and streptomycin on $E$. coli could be vividly understood by observing the selectivity index (SI). All the sulfonamides have selectivity indices ranging from 0.29 for compound $\mathbf{1 8}$ to 0.96 for compound 22 (i.e., less than 1). This implies that streptomycin $(\mathrm{SI}=1)$ was probably more active than any of the sulfonamide scaffolds as regarding the inhibition of E. coli growth. In the same vein, looking through the effect on $S$. aureus, compounds $2,5,17,21$, and 22 were highly active; $4,6,8,10,11,12,14$, and 19 were moderately active; $1,3,7,9,13,15,18$, and 20 exhibited low activity while 16 showed no activity on $S$. aureus (Table 1 ). The comparative study of $\alpha$-toluenesulfonamides to streptomycin on $S$. aureus growth inhibition is worthy, of commendation. From the SI values, compounds $\mathbf{9}, \mathbf{1 2}, \mathbf{1 8}$, and 20 competed favourably with streptomycin while $2,4,5,6,8,13,14,17,19,21$, and 22 ( $\mathrm{SI}=1.08-2.31$ ) showed even a better activity than streptomycin on $S$. aureus. All other compounds exhibited lesser activity than streptomycin $(S I=0.46-0.92)$ on $S$. aureus except 16 which showed no activity.

Due to high zones of inhibition obtained during general sensitivity testing, the minimum inhibitory concentration (MIC) was conducted, first at $100 \mu \mathrm{g} / \mathrm{mL}$ using Russell and Furr method [69]. However, those compounds that could not affect the inhibition of microbial growth at this concentration were further repeated for MIC test at $1000 \mu \mathrm{g} / \mathrm{mL}$. The result of the MIC of this class of compounds on $E$. coli and S. aureus was as shown in Table 2. Interestingly, all the sulfonamides tested showed a concentration-dependent inhibitory effect on the in vitro microbial growth assays [70]. Considering the MIC testing on the gram negative organism (E. coli), it was observed that compounds $1,6,7,11,12,14$, and 22 inhibited the microbial growth at varying values less than or equal to $100 \mu \mathrm{g} / \mathrm{mL}$, whereas, all other compounds were active on $E$. coli at higher concentration (between 125 and $1000 \mu \mathrm{g} / \mathrm{mL}$ ) except 3 and $\mathbf{1 6}$ which had no activity even at $1000 \mu \mathrm{g} / \mathrm{mL}$. Specifically, MIC values of the synthesized compounds on $E$. coli were reported to be $50 \mu \mathrm{g} / \mathrm{mL}$ for 12 and $14 ; 100 \mu \mathrm{g} / \mathrm{mL}$ for 1 and $11 ; 125 \mu \mathrm{g} / \mathrm{mL}$ for $2,4,5,10,17$, and $20 ; 250 \mu \mathrm{g} / \mathrm{mL}$ for $8,9,13,15,19$, and 21 .

Although, the most active sulfonamides on $E$. coli were 6, 7, and 22 with MIC values of 25,25 , and $12.5 \mu \mathrm{g} / \mathrm{mL}$, respectively, none of them could compete with streptomycin (MIC value of $6.25 \mu \mathrm{g} / \mathrm{mL}$ ) in terms of activity. The two rings presence in 22 and their $\pi$ character might be responsible for it being the most active as deduced from the finding of Aissaoui and coworkers [71]. In addition, eight sulfonamides $(2,5,6,8,14,17,21$, and 22) inhibited the $S$. aureus growth at concentration ranging from 1.8 to $100 \mu \mathrm{g} / \mathrm{mL}$. All other compounds were able to affect the expected inhibition 
<smiles>O=C(O)C1CCCN1S(=O)(=O)Cc1ccccc1</smiles><smiles>[R8]C(NS(=O)(=O)Cc1ccccc1)C(=O)O</smiles>

(i) $(\mathrm{COCl})_{2}, \mathrm{DMF}, \mathrm{H}_{2} \mathrm{O}$

(ii) $\mathrm{NH}\left(\mathrm{CH}_{2} \mathrm{CH}_{3}\right)_{2}, \mathrm{Na}_{2} \mathrm{CO}_{3}, \mathrm{H}_{2} \mathrm{O}$ (iii) $-10^{\circ} \mathrm{C}(1 \mathrm{~h}), 0^{\circ} \mathrm{C}(1 \mathrm{~h}), \mathrm{rt}(1 \mathrm{~h})$

(ii) $\mathrm{NH}\left(\mathrm{CH}_{2} \mathrm{CH}_{3}\right)_{2}, \mathrm{NaCO}_{3}, \mathrm{H}_{2} \mathrm{O}$

(iii) $-10^{\circ} \mathrm{C}(1 \mathrm{~h}), 0^{\circ} \mathrm{C}(1 \mathrm{~h}), \mathrm{rt}(1 \mathrm{~h})$

(ii) $\mathrm{NH}\left(\mathrm{CH}_{2} \mathrm{CH}_{3}\right)_{2}, \mathrm{Na}_{2} \mathrm{CO}_{3}, \mathrm{H}_{2} \mathrm{O}$

(iii) $-10^{\circ} \mathrm{C}(1 \mathrm{~h}), 0^{\circ} \mathrm{C}(1 \mathrm{~h}), \mathrm{rt}(1 \mathrm{~h})$

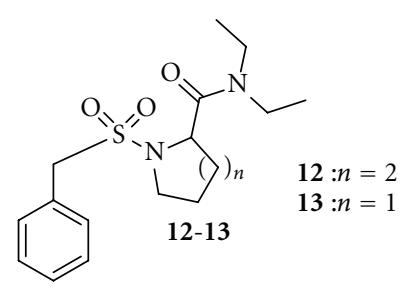<smiles>[R1]C(NS(=O)(=O)Cc1ccccc1)C(=O)N(CC)CC</smiles>

$14: \mathrm{R}_{1}=\mathrm{H}$ (only achiral)

$15: \mathrm{R}_{1}=\mathrm{CH}_{3}$

$16: \mathrm{R}_{1}=\mathrm{CH}_{2} \mathrm{SH}$

$17: \mathrm{R}_{1}=\left(\mathrm{CH}_{2}\right)_{2} \mathrm{SCH}_{3}$<smiles>[R16]C([R3])[C@H](NS(=O)(=O)Cc1ccccc1)C(=O)N(CC)CC</smiles>

$18: \mathrm{R}_{2}=\mathrm{R}_{3}=\mathrm{CH}_{3}$ $19: \mathrm{R}_{2}=\mathrm{CH}_{3}, \mathrm{R}_{3}=\mathrm{OH}$

$20: \mathrm{R}_{2}=\mathrm{H}, \mathrm{R}_{3}=\mathrm{CH}_{2} \mathrm{CONH}_{2}$

$21: \mathrm{R}_{2}=\mathrm{H}, \mathrm{R}_{3}=\mathrm{Ph}$

$22: \mathrm{R}_{2}=\mathrm{H}, \mathrm{R}_{3}=\mathrm{PhCH}_{2} \mathrm{OSO}_{2-}$

SCHeme 3: Synthesis of $N, N$-diethylamide substituted $\alpha$-tolylsulfonamides, $12-22$.

from $125 \mu \mathrm{g} / \mathrm{mL}$ to $1000 \mu \mathrm{g} / \mathrm{mL}$ except 16 which had no activity even at $1000 \mu \mathrm{g} / \mathrm{mL}$. The significant antibacterial activity of the synthesized compounds may be explained by the ability of its sulfonamide binding site to mimic $p$ aminobenzoic acid (PABA) which is an essential growth factor in the targeted organisms as earlier documented in the literatures [14-16]. The explanation for this encouraging activity of the synthesized sulfonamides could be traceable to the mode of action of sulfonamide drugs. This is based on the inhibition of DNA synthesis [72] by interfering with para-aminobenzoic acid (PABA) in biosynthesis of folic acid [73].

Furthermore, from the structure activity relationship (SAR) study, it was observed that the nature of side chains $\left(\mathrm{R}_{1}\right.$ and $\mathrm{R}_{2}-\mathrm{CH}-\mathrm{R}_{3}$ ) of the $\alpha$-tolylsulfonamides and the presence of $\mathrm{N}, \mathrm{N}$-diethylated amido moieties $\left\{\left(\mathrm{CH}_{3} \mathrm{CH}_{2}\right)_{2} \mathrm{~N}-\right.$ $\mathrm{C}=\mathrm{O}\}$ of the amide contributed immensely toward synergistic or antagonistic effect on the reported in vitro antibacterial activity. For instance, the antibacterial activity of structurally related $\alpha$-tolylsulfonamides containing $\mathrm{R}_{1}=\left(\mathrm{CH}_{2}\right)_{2} \mathrm{SCH}_{3}$, 6; $\mathrm{R}_{2}=\mathrm{R}_{3}=\mathrm{CH}_{3}, 7$, on $E$. coli were very high with MIC value of $25 \mathrm{ppm}$ whereas the activity reduced to moderate when $\mathrm{R}_{1}$ $=\mathrm{CH}_{3}, \mathbf{4} ; \mathrm{R}_{1}=\mathrm{CH}_{2} \mathrm{SH}, 5$ with MIC value of $125 \mathrm{ppm}$. The synergistic effect due to the presence of $\mathrm{N}, \mathrm{N}$-diethylamido moieties was noticed in some synthetic conversions such as 1 $\rightarrow 12,3 \rightarrow 14$ and $11 \rightarrow 22$. Thus, $\alpha$-tolylsulfonamides 1 and 11 had moderate activity on E. coli while their corresponding diethylamido $\mathbf{1 2}$ and $\mathbf{2 2}$ had better and higher activity on E. coli. In fact, $\alpha$-tolylsulfonamide 3 had no activity on $E$. coli whereas its $N, N$-diethyl amidated counterpart, 14 had very high activity with MIC value of $50 \mathrm{ppm}$. The synergistic effect noticed herein might be as a result of electron donating nature of the $\mathrm{R}_{1}$ side chain. On the contrary, antagonistic effect was observed in the synthetic modification of $\mathbf{5} \rightarrow \mathbf{1 6}$ and $\mathbf{6} \rightarrow \mathbf{1 7}$ as far as presence of $N, N$-diethyl-substituted amido moieties was concerned. This reverse order of activity on $E$. coli might be as a result of the presence of sulfur atom in $\mathbf{5}$ and $\mathbf{6}$ which might have altered the electron donating prowess. Considering the structural relationship effect on the activity of the synthesized compounds against $S$. aureus, it was discovered that $N, N$-diethyl-substituted amides 14, 17, 21, and 22 had better activity than their $\alpha$-tolylsulfonamide precursors 3, 6, 10, and 11 .

\section{Conclusion}

It was discovered that aqueous medium approach at ambient temperature used here-in was a highly efficient procedure for the preparation of various $\alpha$-tolylsulfonamide derivatives 1-11 in good to excellent yield. The synthetic modification of these derivatives as precursors to furnish disubstituted amide bearing sulfonamides $\mathbf{1 2 - 2 2}$ was also successfully achieved. The antibacterial evaluation study showed 1-(benzylsulfonyl) pyrrolidine-2-carboxylic acid, 2 to be the most a ctive sulfonamide on S. aureus while 4-(3-(diethylamino)-3oxo-2-(phenylmethylsulfonamido)propyl) phenylphenylmethane sulfonate, 22 emerged as the most active on the growth inhibition of E. coli. Thus, this work will be very useful for further studies in terms of toxicity effect and structural activity relationship (SAR) study by using various types of 
TABLE 1: Result of general sensitivity testing with zones of inhibitory in $(\mathrm{mm})$ and selectivity index.

\begin{tabular}{|c|c|c|c|c|}
\hline \multirow{4}{*}{ Compd. no. } & \multicolumn{4}{|c|}{ In vitro antibacterial activity } \\
\hline & \multicolumn{4}{|c|}{ Organisms } \\
\hline & \multicolumn{2}{|c|}{ E. coli ATCC 25922} & \multicolumn{2}{|c|}{ S. aureus ATCC 6538} \\
\hline & $\mathrm{ZOI}(\mathrm{mm})$ & SI & $\mathrm{ZOI}(\mathrm{mm})$ & SI \\
\hline 1 & ++ & 0.61 & + & 0.92 \\
\hline 2 & ++ & 0.54 & +++ & 2.31 \\
\hline 3 & - & - & + & 0.69 \\
\hline 4 & ++ & 0.57 & ++ & 1.08 \\
\hline 5 & ++ & 0.57 & +++ & 2.15 \\
\hline 6 & +++ & 0.86 & ++ & 1.15 \\
\hline 7 & +++ & 0.89 & + & 0.46 \\
\hline 8 & + & 0.46 & ++ & 1.15 \\
\hline 9 & + & 0.36 & + & 1.00 \\
\hline 10 & ++ & 0.57 & ++ & 0.92 \\
\hline 11 & ++ & 0.75 & ++ & 0.92 \\
\hline 12 & +++ & 0.84 & ++ & 1.00 \\
\hline 13 & + & 0.43 & + & 1.08 \\
\hline 14 & +++ & 0.82 & ++ & 1.38 \\
\hline 15 & + & 0.36 & + & 0.92 \\
\hline 16 & - & - & - & - \\
\hline 17 & ++ & 0.57 & +++ & 2.23 \\
\hline 18 & + & 0.29 & + & 1.00 \\
\hline 19 & + & 0.43 & ++ & 1.08 \\
\hline 20 & ++ & 0.54 & + & 1.00 \\
\hline 21 & + & 0.39 & +++ & 2.00 \\
\hline 22 & +++ & 0.96 & +++ & 2.00 \\
\hline Str. & +++ & 1.00 & ++ & 1.00 \\
\hline
\end{tabular}

+: Less active $5-12 \mathrm{~mm}$; ++: moderately active $13-19 \mathrm{~mm}$; +++: highly active $20-31 \mathrm{~mm}$; -: resistance; str.: streptomycin clinical reference; Z.O.I.: zone of inhibition; S.I.: selective index obtained by comparing inhibition zone of compound to that of streptomycin standard; E. coli: Escherichia coli (ATCC 25922) ${ }^{\mathrm{G}-}$; S. aureus: Staphylococcus aureus (ATCC 6538) ${ }^{\mathrm{G}+} ; \mathrm{G}-$ : gram negative; $\mathrm{G}+$ : gram positive.

dialkylated substituents to monitor the trend of improvement on their biological and pharmacological properties.

\section{Experimental}

4.1. General Conditions. The ${ }^{1} \mathrm{H}-\mathrm{NMR}$ spectra were recorded in $\mathrm{D}_{2} \mathrm{O}$ on NMR Bruker DPX 400 spectrometer operating at $400 \mathrm{MHz}$. TMS was used as an internal standard with the deuterium signal of the solvent as the lock and chemical shifts $\delta$ recorded in ppm. The ${ }^{13} \mathrm{C}$-NMR spectra were run in addition of a few drops of Dioxane but at $100 \mathrm{MHz}$ frequency. The melting points were determined on XT4 Digital Binocular Microscope melting point apparatus manufactured by Beijing Technical Instrument Co. Ltd. and were uncorrected. IR spectra were run on Varian Excalibur HE 3100 FT-IR Spectrometer while the mass spectra were obtained using Waters GCT Premier Spectrometer. The elemental analyses $(\mathrm{C}, \mathrm{H}$, and $\mathrm{N})$ of the compounds were performed using Flash EA 1112 Elemental Analyzer.
Lyophilization was carried out where necessary by using FD1 Freeze Drier while concentration and removal of solvents were achieved with RE-2000B Rotary Evaporator.

In addition, the $\mathrm{pH}$ was monitored and confirmed during acidification by using Portable pH Meter Model PHB4. All drying was conducted at a reduced pressure with DHG9023A Vacuum Oven. The reaction progress was monitored with TLC using $\mathrm{CHCl}_{3} / \mathrm{CH}_{3} \mathrm{OH}$ solvent system and the developed plates were visualized under UV lamp and/or in iodine tank where necessary. Column chromatographic purifications were carried out on Merck silica gel F (Mesh 200-300). Organic solutions were dried over anhydrous $\mathrm{Na}_{2} \mathrm{SO}_{4}$ and concentrated with a Buchi Rotary Evaporator at reduced pressure. At all stages of the experiments, the synthetic protocols were effected in bone dried solvents under nitrogen atmosphere in dried glassware which were wiped with stream flow of nitrogen gas prior to use and $\mathrm{SOCl}_{2}$ was freshly distilled prior to use. Other reagents were used directly after ascertaining the purity condition.

\subsection{Synthesis}

4.2.1. General Procedure of $\alpha$-Tolylsulfonamide Derivatives 111. To a solution of L-amino acid ( $5 \mathrm{mmol})$ in $\mathrm{H}_{2} \mathrm{O}(6 \mathrm{~mL})$ was added $\mathrm{Na}_{2} \mathrm{CO}_{3}(1.113 \mathrm{~g}, 10.5 \mathrm{mmol})$ with a continuous stirring until all the solutes had dissolved. The clear solution was cooled to $-10^{\circ} \mathrm{C}$ and $\alpha$-toluenesulfonyl chloride, $\alpha$ $\mathrm{TsCl}(1.144 \mathrm{~g}, 6 \mathrm{mmol})$ was added in three batches over a period of $1 \mathrm{~h}$. It was warmed up to $0^{\circ} \mathrm{C}$ and stirred there for $1 \mathrm{~h}$. Finally, the reacting mixture was then warmed up to room temperature and allowed to stir there for $48 \mathrm{~h}$. The reaction was quenched by the addition of DCM $(10 \mathrm{~mL})$ and transferred into separatory funnel where the excess of $\alpha$-TsCl was removed by extraction. The aqueous layer was then worked up to give a clear solution by the addition of $2 \mathrm{~N} \mathrm{HCl}$ until the $\mathrm{pH} 2.2$ was attained. The clear liquid was then lyophilized at $-52^{\circ} \mathrm{C}$ under reduced pressure for $12 \mathrm{~h}$ to obtain the crude solid product which was purified by column chromatography $\left(\mathrm{CHCl}_{3} / \mathrm{CH}_{3} \mathrm{OH}, 3: 1\right)$ to afford $\alpha$-toluene sulfonamides $\mathbf{1}-\mathbf{1 1}$ in excellent yields.

(1) 1-(Benzylsulfonyl)piperidine-2-carboxylic Acid, 1. Reagents: L-pipecolic acid, yield $98 \%, \operatorname{mp} 248^{\circ} \mathrm{C}$ ( dec), $R_{f}=0.87$. ${ }^{1} \mathrm{H}-\mathrm{NMR}\left(\mathrm{D}_{2} \mathrm{O}, 400 \mathrm{MHz}\right) \delta: 7.52(\mathrm{~s}, 5 \mathrm{H}, \mathrm{Ar}-\mathrm{H}), 4.28(\mathrm{~s}$, $\left.2 \mathrm{H}, \mathrm{CH}_{2}-\mathrm{SO}_{2}\right), 4.08-4.02\left(\mathrm{dd}, J_{1}=3.44 \mathrm{~Hz}, J_{2}=15.12 \mathrm{~Hz}\right.$, $\left.1 \mathrm{H}, \mathrm{HOOC}-\mathrm{CH}-\mathrm{CH}_{2}\right), 3.57-3.53\left(\mathrm{~m}, 1 \mathrm{H}, \mathrm{CHa}\right.$ of $\left.\mathrm{CH}_{2}-\mathrm{N}\right)$, $3.16-3.10\left(\mathrm{~m}, 1 \mathrm{H}, \mathrm{CHb}\right.$ of $\left.\mathrm{CH}_{2}-\mathrm{N}\right), 2.40-2.36(\mathrm{~m}, 1 \mathrm{H}, \mathrm{CH})$, 2.01-1.92 (m, 2H, $2 \times \mathrm{CH}), 1.81-1.68\left(\mathrm{~m}, 3 \mathrm{H}, \mathrm{CH} \& \mathrm{CH}_{2}\right)$ ppm. ${ }^{13} \mathrm{C}-\mathrm{NMR}$ (Dioxane, $\left.100 \mathrm{MHz}\right) \delta: 172.5$ (CO), 132.5, 131.2 (2CH aromatic), 129.5 (2CH aromatic), 128.9, 57.7, 57.6, 44.8, 26.5, $22.1\left(2 \mathrm{CH}_{2}\right)$ ppm. IR $(\mathrm{KBr}) \mathrm{cm}^{-1}: 3422(\mathrm{OH}$ of acid), 2974 ( $\mathrm{CH}$ aromatic), 2822 ( $\mathrm{CH}$ aliphatic), 1736 ( $\mathrm{C}=\mathrm{O}$ of $\mathrm{COOH}), 1603$ ( $\mathrm{C}=\mathrm{C}), 1238,1159$ ( $\mathrm{SO}_{2}$ two bands), 700 (Ar-H). MS: in m/z [rel. \%]: $269.1\left[\mathrm{M}^{+}-\mathrm{CH}_{2}, 3.2 \%\right]$, 180.1 [55\%], 179.1 [65\%], $178.1\left[\mathrm{M}^{+}-\mathrm{PhCH}_{2}, 100 \%\right], 165.1$ [30\%], 121.0 [42\%], $77.0\left[\mathrm{Ph}^{+}, 13 \%\right], 64.0\left[\mathrm{SO}_{2}^{+}, 31.7 \%\right]$. Anal. Calcd. for $283.35 \mathrm{C}_{13} \mathrm{H}_{17} \mathrm{NO}_{4} \mathrm{~S}$ : C, 55.11; H, 6.05; N, 4.94. Found: C, 55.29; H, 5.94; N, 4.86. 
TABLE 2: Result of MIC test of $\alpha$-tolylsulfonamide on targeted organisms ( $\mu \mathrm{g} / \mathrm{mL})$.

\begin{tabular}{|c|c|c|c|c|}
\hline \multirow{4}{*}{ Compd. no. } & \multicolumn{4}{|c|}{ Minimum inhibitory concentration $(\mu \mathrm{g} / \mathrm{mL})$} \\
\hline & \multicolumn{4}{|c|}{ Organisms } \\
\hline & \multicolumn{2}{|c|}{ E. coli ATCC 25922} & \multicolumn{2}{|c|}{ S. aureus ATCC 6538} \\
\hline & at $100 \mu \mathrm{g} / \mathrm{mL}$ & at $1000 \mu \mathrm{g} / \mathrm{mL}$ & at $100 \mu \mathrm{g} / \mathrm{mL}$ & at $1000 \mu \mathrm{g} / \mathrm{mL}$ \\
\hline 1 & 100 & $<1000$ & $>100$ & 250 \\
\hline 2 & $>100$ & 125 & 1.8 & $<1000$ \\
\hline 3 & $>100$ & - & $>100$ & 250 \\
\hline 4 & $>100$ & 125 & $>100$ & 125 \\
\hline 5 & $>100$ & 125 & 50 & $<1000$ \\
\hline 6 & 25 & $<1000$ & 100 & $<1000$ \\
\hline 7 & 25 & $<1000$ & $>100$ & 500 \\
\hline 8 & $>100$ & 250 & 100 & $<1000$ \\
\hline 9 & $>100$ & 250 & $>100$ & 250 \\
\hline 10 & $>100$ & 125 & $>100$ & 125 \\
\hline 11 & 100 & $<1000$ & $>100$ & 125 \\
\hline 12 & 50 & $<1000$ & $>100$ & 125 \\
\hline 13 & $>100$ & 250 & $>100$ & 250 \\
\hline 14 & 50 & $<1000$ & 62.5 & $<1000$ \\
\hline 15 & $>100$ & 250 & $>100$ & 1000 \\
\hline 16 & $>100$ & - & $>100$ & - \\
\hline 17 & $>100$ & 125 & 25 & $<1000$ \\
\hline 18 & $>100$ & 500 & $>100$ & 1000 \\
\hline 19 & $>100$ & 250 & $>100$ & 125 \\
\hline 20 & $>100$ & 125 & $>100$ & 250 \\
\hline 21 & $>100$ & 250 & 25 & $<1000$ \\
\hline 22 & 12.5 & $<1000$ & 25 & $<1000$ \\
\hline Str. & 6.25 & $<1000$ & $>100$ & 125 \\
\hline
\end{tabular}

$>100$ means that if there was no growth inhibition at $100 \mu \mathrm{g} / \mathrm{mL}$, it was repeated at $1000 \mu \mathrm{g} / \mathrm{mL},<1000 \mu \mathrm{g} / \mathrm{mL}$ means that growth inhibition has already been experienced at lower concentration less than or equal to $100 \mu \mathrm{g} / \mathrm{mL}$; hence, there is no need to repeat the test at $1000 \mu \mathrm{g} / \mathrm{mL}$. — means no activity was observed even at $1000 \mu \mathrm{g} / \mathrm{mL}$. Str. means streptomycin clinical reference.

(2) 1-(Benzylsulfonyl)pyrrolidine-2-carboxylic Acid, 2. L-amino acid is L-proline; yield $1.24 \mathrm{~g}(92 \%) ; \mathrm{mp} 108-110^{\circ} \mathrm{C} ; R_{f}$ $=0.84 .{ }^{1} \mathrm{H}-\mathrm{NMR}\left(\mathrm{D}_{2} \mathrm{O}, 400 \mathrm{MHz}\right) \delta: 7.47(\mathrm{~s}, 5 \mathrm{H}, \mathrm{Ar}-\mathrm{H})$, $4.46-4.43\left(\mathrm{dd}, J_{1}=7.2 \mathrm{~Hz}, J_{2}=15.76 \mathrm{~Hz}, 1 \mathrm{H}, \mathrm{HOOC}-\mathrm{CH}-\right.$ $\left.\mathrm{CH}_{2}(\mathrm{a}, \mathrm{b})\right), 4.24\left(\mathrm{~s}, 2 \mathrm{H}, \mathrm{CH}_{2}-\mathrm{SO}_{2}\right), 3.48-3.45(\mathrm{t}, J=7.28 \mathrm{~Hz}$, $\left.2 \mathrm{H}, \mathrm{N}-\mathrm{CH}_{2}-\mathrm{CH}_{2}\right), 2.46-2.45\left(\mathrm{~m}, 1 \mathrm{H}, \mathrm{CHa}\right.$ of $\left.\mathrm{CH}_{2}\right), 2.22-2.21$ $\left(\mathrm{m}, 1 \mathrm{H}, \mathrm{CHb}\right.$ of $\mathrm{CH}_{2}$ ), 2.15-2.09 (quintet, $J=6.8 \mathrm{~Hz}, 2 \mathrm{H}$, $\left.\mathrm{CH}_{2}-\mathrm{CH}_{2}-\mathrm{CH}_{2}(\mathrm{a}, \mathrm{b})\right)$ ppm. ${ }^{13} \mathrm{C}-\mathrm{NMR}$ (Dioxane, $100 \mathrm{MHz}$ ) $\delta$ : $173.1(\mathrm{CO}), 132.6,131.2(2 \mathrm{CH}$ aromatic), $129.5(2 \mathrm{CH}$ aromatic), $128.9,60.6,57.7,47.1,29.2,24.3 \mathrm{ppm}$. IR (KBr) $\mathrm{cm}^{-1}: 3441$ (OH of acid), $2980(\mathrm{CH}$ aromatic), $2828(\mathrm{CH}$ aliphatic), $1728(\mathrm{C}=\mathrm{O}$ of $\mathrm{COOH}), 1620(\mathrm{C}=\mathrm{C}), 1219,1151$ ( $\mathrm{SO}_{2}$ two bands), 700 (Ar-H). MS: in $\mathrm{m} / z$ [rel. \%]: 270.1 $\left[\mathrm{MH}^{+}, 6.5 \%\right], 269.1\left[\mathrm{M}^{+}, 9 \%\right], 179.1[18.4 \%], 178.1\left[\mathrm{M}^{+}-\right.$ $\mathrm{PhCH}_{2}, 100 \%$ ], 176.1 [32.4\%], 122.0 [49\%], 105.0 [32\%]. Anal. Calcd. for $269.32 \mathrm{C}_{12} \mathrm{H}_{15} \mathrm{NO}_{4} \mathrm{~S}$ : C, 53.52; H, 5.61; N, 5.20. Found: C, 53.77; H, 5.49; N, 5.34.

(3) 2-(Phenylmethylsulfonamido)acetic Acid, 3. Amino acid is glycine; yield $1.08 \mathrm{~g}(94 \%) ; \mathrm{mp} 150-151^{\circ} \mathrm{C} ; R_{f}=0.51$. ${ }^{1} \mathrm{H}-\mathrm{NMR}\left(\mathrm{D}_{2} \mathrm{O}, 400 \mathrm{MHz}\right) \delta: 7.45$ (s, 5H, Ar-H), 4.19 (s, $2 \mathrm{H}, \mathrm{CH}_{2}-\mathrm{SO}_{2}$ ), 3.76 (s, $\left.2 \mathrm{H}, \mathrm{CH}_{2}-\mathrm{COOH}\right)$ ppm. ${ }^{13} \mathrm{C}-\mathrm{NMR}$
(Dioxane, $100 \mathrm{MHz})$ 8: 170.6 (CO), 132.7, $131.2(2 \mathrm{CH}$ aromatic), 129.5 (2CH aromatic), 128.9, 57.7, $40.8 \mathrm{ppm}$. IR $(\mathrm{KBr}) \mathrm{cm}^{-1}: 3433(\mathrm{OH}$ of acid), $3030(\mathrm{~N}-\mathrm{H}), 2990(\mathrm{CH}$ aromatic), 2832 ( $\mathrm{CH}$ aliphatic), $1736(\mathrm{C}=\mathrm{O}$ of $\mathrm{COOH}), 1616$ $(\mathrm{C}=\mathrm{C}), 1215,1171\left(\mathrm{SO}_{2}\right.$ two bands), $702(\mathrm{Ar}-\mathrm{H})$. MS: in $\mathrm{m} / \mathrm{z}$ [rel. \%]: 212.1 [M+-OH, 7.9\%], 180.1 [73\%], 179.1 [88\%], $178.1\left[\mathrm{M}^{+}-\mathrm{PhCH}_{2}, 100 \%\right], 91.1\left[\mathrm{PhCH}_{2}^{+}, 48 \%\right], 64$ [26\%] $45\left[{ }^{+} \mathrm{COOH}, 2.4 \%\right]$. Anal. Calcd. for $229.26 \mathrm{C}_{9} \mathrm{H}_{11} \mathrm{NO}_{4} \mathrm{~S}: \mathrm{C}$, 47.15; H, 4.84; N, 6.11. Found: C, 46.90; H, 5.01; N, 5.97.

(4) 2-(Phenylmethylsulfonamido)propanoic Acid, 4. L-amino acid is L-alanine; yield $1.18 \mathrm{~g}(97 \%) ; \mathrm{mp} 126-128^{\circ} \mathrm{C} ; R_{f}$ $=0.81 .{ }^{1} \mathrm{H}-\mathrm{NMR}\left(\mathrm{D}_{2} \mathrm{O}, 400 \mathrm{MHz}\right) \delta: 7.47(\mathrm{~s}, 5 \mathrm{H}, \mathrm{Ar}-\mathrm{H})$, $4.22\left(\mathrm{~s}, 2 \mathrm{H}, \mathrm{CH}_{2}-\mathrm{SO}_{2}\right), 4.14-4.08(\mathrm{q}, J=7.28 \mathrm{~Hz}, 1 \mathrm{H}$, $\left.\mathrm{CH}-\mathrm{CH}_{3}\right), 1.60-1.58\left(\mathrm{~d}, J=7.28 \mathrm{~Hz}, 3 \mathrm{H}, \mathrm{CH}_{3}-\mathrm{CH}\right) \mathrm{ppm}$. ${ }^{13}$ C-NMR (Dioxane, $100 \mathrm{MHz}$ ) $\delta: 176.8$ (CO), 131.0, 130.6 (2CH aromatic), 129.3 (2CH aromatic), 129.1, 60.4, 52.1, $19.6 \mathrm{ppm}$. IR ( $\mathrm{KBr}) \mathrm{cm}^{-1}: 3424(\mathrm{OH}$ of acid), $2974(\mathrm{CH}$ aromatic), 2822 ( $\mathrm{CH}$ aliphatic), $1751(\mathrm{C}=\mathrm{O}$ of $\mathrm{COOH}), 1599$ $(\mathrm{C}=\mathrm{C}), 1213,1169\left(\mathrm{SO}_{2}\right.$ two bands $), 698(\mathrm{Ar}-\mathrm{H})$. MS: in $\mathrm{m} / \mathrm{z}$ [rel. \%]: 212.1 [22\%], 180.1 [81.5\%], 179.1 [91\%], 178.1 [85\%], 165.1 [M- $\left.\mathrm{PhCH}_{3}, 55 \%\right], 122.0$ [80\%], 121.0 [100\%], 
$77.0\left[\mathrm{Ph}^{+}, 71.4 \%\right], 64.0\left[\mathrm{SO}_{2}^{+}, 54.6 \%\right], 51.0$ [28\%]. Anal. Calcd. for $243.28 \mathrm{C}_{10} \mathrm{H}_{13} \mathrm{NO}_{4} \mathrm{~S}$ : C, 49.37; H, 5.39; N, 5.76. Found: C, 49.29; H, 5.28; N, 5.94.

(5) 3-Mercapto-2-(phenylmethylsulfonamido)propanoic Acid, 5. L-amino acid is cysteine; yield $1.22 \mathrm{~g}$ (89\%); $\mathrm{mp} 171-$ $173^{\circ} \mathrm{C} ; R_{f}=0.49 .{ }^{1} \mathrm{H}-\mathrm{NMR}\left(\mathrm{D}_{2} \mathrm{O}, 400 \mathrm{MHz}\right) \delta: 7.49(\mathrm{~s}$, $5 \mathrm{H}, \mathrm{Ar}-\mathrm{H}), 4.50-4.47\left(\mathrm{dd}, J_{1}=4.24 \mathrm{~Hz}, J_{2}=7.92 \mathrm{~Hz}, 1 \mathrm{H}\right.$, $\left.\mathrm{CH}_{2}-\mathrm{CH}-\mathrm{COOH}\right), 4.24$ (s, 2H, $\mathrm{CH}_{2}-\mathrm{SO}_{2}$ ), 3.55-3.50 (dd, $J_{1}=4.24 \mathrm{~Hz}, J_{2}=20 \mathrm{~Hz}, 1 \mathrm{H}, \mathrm{CHa}$ of $\left.\mathrm{CH}_{2}-\mathrm{CH}\right), 3.39-3.33$ $\left(\mathrm{dd}, J_{1}=7.92 \mathrm{~Hz}, J_{2}=20 \mathrm{~Hz}, 1 \mathrm{H}, \mathrm{CHb}\right.$ of $\left.\mathrm{CH}_{2}-\mathrm{CH}\right) \mathrm{ppm}$. ${ }^{13} \mathrm{C}-\mathrm{NMR}$ (Dioxane, $\left.100 \mathrm{MHz}\right) \delta: 171.5$ (CO), 132.6, 131.3 (2 $\mathrm{CH}$ aromatic), 129.5 (2CH aromatic), 128.7, 57.8, 52.7, 37.2 ppm. IR (KBr) cm ${ }^{-1}: 3439(\mathrm{OH}$ of acid), $2978(\mathrm{CH}$ aromatic), 2832 ( $\mathrm{CH}$ aliphatic), $1728(\mathrm{C}=\mathrm{O}$ of $\mathrm{COOH}), 1618$ (C=C), 1207, 1159 ( $\mathrm{SO}_{2}$ two bands), 810 (Ar-H). MS: in $m / z$ [rel. \%]: 214.1 [31.7\%], 123.0 [100\%], 122.0 [90\%], 92.1 $\left[\mathrm{PhCH}_{3}^{+}, 33 \%\right], 91.0\left[\mathrm{PhCH}_{2}^{+}, 88 \%\right], 77.0\left[\mathrm{Ph}^{+}, 8 \%\right], 65.0$ [ $\left.\mathrm{HSO}_{2}^{+}, 34 \%\right], 45.0\left[{ }^{+} \mathrm{COOH}, 28 \%\right], 36.0$ [34\%]. Anal. Calcd. for $275.35 \mathrm{C}_{10} \mathrm{H}_{13} \mathrm{NO}_{4} \mathrm{~S}_{2}$ : C, 43.62; $\mathrm{H}, 4.76 ; \mathrm{N}, 5.09$. Found: C, 43.45; H, 4.94; N, 5.07.

(6) 4-(Methylthio)-2-(phenylmethylsulfonamido)butanoic Acid, 6. L-amino acid is L-methionine; yield $1.33 \mathrm{~g}(87.7 \%)$; $\operatorname{mp} 89-90^{\circ} \mathrm{C} ; R_{f}=0.68 .{ }^{1} \mathrm{H}-\mathrm{NMR}\left(\mathrm{D}_{2} \mathrm{O}, 400 \mathrm{MHz}\right) \delta: 7.47(\mathrm{~s}$, $5 \mathrm{H}, \mathrm{Ar}-\mathrm{H}), 4.23$ (s, $\left.2 \mathrm{H}, \mathrm{CH}_{2}-\mathrm{SO}_{2}\right), 4.01-3.97(\mathrm{t}, J=8.84 \mathrm{~Hz}$, $\left.1 \mathrm{H}, \mathrm{CH}-\mathrm{CH}_{2}\right), 2.76-2.72\left(\mathrm{t}, \mathrm{J}=7.40 \mathrm{~Hz}, 2 \mathrm{H}, \mathrm{CH}_{2}-\mathrm{CH}_{2}-\mathrm{S}\right)$, 2.33-2.29 (m, 1H, CH), 2.28-2.21 (m, 1H, CH), 2.19 (s, 3H, $\mathrm{CH}_{3}-\mathrm{S}$ ) ppm. ${ }^{13} \mathrm{C}-\mathrm{NMR}$ (Dioxane, $\left.100 \mathrm{MHz}\right) \delta: 172.9$ (CO), 132.6, 131.2 (2CH aromatic), 129.5 (2CH aromatic), 128.7, 57.9, 52.8, 29.8, 29.4, 14.7 ppm. IR $(\mathrm{KBr}) \mathrm{cm}^{-1}: 3442(\mathrm{OH}$ of acid), 2974 ( $\mathrm{CH}$ aromatic), 2833 ( $\mathrm{CH}$ aliphatic), 2774 (CH aliphatic), 1742 ( $\mathrm{C}=\mathrm{O}$ of $\mathrm{COOH}), 1590(\mathrm{C}=\mathrm{C}), 1211$, 1161 ( $\mathrm{SO}_{2}$ two bands), 698 (Ar-H). Anal. Calcd. for 303.40 $\mathrm{C}_{12} \mathrm{H}_{17} \mathrm{NO}_{4} \mathrm{~S}_{2}$ : C, 47.51; H, 5.65; N, 4.62. Found: C, 47.49; $\mathrm{H}, 5.64 ; \mathrm{N}, 4.66$.

(7) 3-Methyl-2-(phenylmethylsulfonamido)butanoic Acid, 7. L-amino acid is L-valine; yield $1.34 \mathrm{~g}$ (98.7\%); $\mathrm{mp} 137$ $138^{\circ} \mathrm{C} ; R_{f}=0.83 .{ }^{1} \mathrm{H}-\mathrm{NMR}\left(\mathrm{D}_{2} \mathrm{O}, 400 \mathrm{MHz}\right) \delta: 7.49(\mathrm{~s}, 5 \mathrm{H}$, Ar-H), 4.25 (s, 2H, CH $-\mathrm{SO}_{2}$ ), 4.04-4.03 (d, $J=4.4 \mathrm{~Hz}, 1 \mathrm{H}$, $\mathrm{CH}-\mathrm{CH}-\mathrm{COOH}), 2.47-2.38$ (m, 1H, CH-CH- $\left.\left(\mathrm{CH}_{3}\right)_{2}\right), 1.15-$ $1.13\left(\mathrm{~d}, J=7.04 \mathrm{~Hz}, 3 \mathrm{H}, \mathrm{CH}_{3}-\mathrm{CH}\right), 1.13-1.11$ (d, $J=7.08 \mathrm{~Hz}$, $3 \mathrm{H}, \mathrm{CH}_{3}-\mathrm{CH}$ ) ppm. ${ }^{13} \mathrm{C}-\mathrm{NMR}$ (Dioxane, $\left.100 \mathrm{MHz}\right) \delta: 172.4$ (CO), $132.5,131.2$ (2CH aromatic), 129.5 (2CH aromatic), $128.9,59.2,57.7,29.8,18.1,17.8$ ppm. IR (KBr) cm ${ }^{-1}: 3447$ ( $\mathrm{OH}$ of acid), 2974 ( $\mathrm{CH}$ aromatic), 2833 ( $\mathrm{CH}$ aliphatic), $2783(\mathrm{CH}$ aliphatic), $1730(\mathrm{C}=\mathrm{O}$ of $\mathrm{COOH}), 1618(\mathrm{C}=\mathrm{C})$, 1225, 1165 ( $\mathrm{SO}_{2}$ two bands), 700 (Ar-H). MS: in $\mathrm{m} / z$ [rel. \%]: $271.1\left[\mathrm{M}^{+}, 14 \%\right], 91.0\left[\mathrm{PhCH}_{2}^{+}, 30 \%\right], 75.0$ [65\%], 72.1 [100\%], 55.0 [79\%], 29.0 [50\%]. Anal. Calcd. for 271.34 $\mathrm{C}_{12} \mathrm{H}_{17} \mathrm{NO}_{4} \mathrm{~S}$ : C, 53.12; H, 6.32; N, 5.16. Found: C, 53.31; H, $6.50 ; \mathrm{N}, 5.20$.

(8) 3-Hydroxy-2-(phenylmethylsulfonamido)butanoic Acid, 8. L-amino acid is L-threonine; yield $1.25 \mathrm{~g}$ (91.5\%); mp 194$195^{\circ} \mathrm{C} ; R_{f}=0.48 .{ }^{1} \mathrm{H}-\mathrm{NMR}\left(\mathrm{D}_{2} \mathrm{O}, 400 \mathrm{MHz}\right) \delta: 7.49(\mathrm{~s}, 5 \mathrm{H}$,
Ar-H), 4.48-4.46 (m, 1H, CH), 4.24 (s, 2H, $\left.\mathrm{CH}_{2}-\mathrm{SO}_{2}\right)$, 4.05$4.04(\mathrm{~d}, \mathrm{~J}=4.4 \mathrm{~Hz}, 1 \mathrm{H}, \mathrm{CH}-\mathrm{CH}-\mathrm{COOH}), 1.42-1.41$ (d, $J=$ $6.64 \mathrm{~Hz}, 3 \mathrm{H}, \mathrm{CH}_{3}-\mathrm{CH}$ ) ppm. ${ }^{13} \mathrm{C}-\mathrm{NMR}$ (Dioxane, $100 \mathrm{MHz}$ ) $\delta$ : $171.5(\mathrm{CO}), 132.5,131.2(2 \mathrm{CH}$ aromatic $), 129.5(2 \mathrm{CH}$ aromatic), 128.9, 66.1, 59.4, 57.7, $19.8 \mathrm{ppm}$. IR (KBr) $\mathrm{cm}^{-1}$ : 3404 (OH of acid), 2976 ( $\mathrm{CH}$ aromatic), 2824 ( $\mathrm{CH}$ aliphatic), $1740(\mathrm{C}=\mathrm{O}$ of $\mathrm{COOH}), 1601(\mathrm{C}=\mathrm{C}), 1219,1157\left(\mathrm{SO}_{2}\right.$ two bands), 700 (Ar-H). Anal. Calcd. for $273.31 \mathrm{C}_{11} \mathrm{H}_{15} \mathrm{NO}_{5} \mathrm{~S}: \mathrm{C}$, 48.34; H, 5.53; N, 5.12. Found: C, 48.29; H, 5.61; N, 4.98.

(9) 5-Amino-5-oxo-2-(phenylmethylsulfonamido)pentanoic Acid, 9. L-amino acid is L-glutamine; yield $1.41 \mathrm{~g}$ (94.1\%); $\operatorname{mp} 211-214^{\circ} \mathrm{C} ; R_{f}=0.38 .{ }^{1} \mathrm{H}-\mathrm{NMR}\left(\mathrm{D}_{2} \mathrm{O}, 400 \mathrm{MHz}\right) \delta: 7.48$ (s, 5H, Ar-H), 4.47-4.43 (dd, $J_{1}=5.04 \mathrm{~Hz}, J_{2}=14.32 \mathrm{~Hz}$, $\left.1 \mathrm{H}, \mathrm{HOOC}-\mathrm{CH}-\mathrm{CH}_{2}\right), 4.23\left(\mathrm{~s}, 2 \mathrm{H}, \mathrm{CH}_{2}-\mathrm{SO}_{2}\right), 2.62-2.55$ $(\mathrm{m}, 1 \mathrm{H}, \mathrm{CH}), 2.49-2.44\left(\mathrm{dd}, J_{1}=9.2 \mathrm{~Hz}, J_{2}=18.72 \mathrm{~Hz}, 2 \mathrm{H}\right.$, $\left.\mathrm{CH}_{2}-\mathrm{CH}_{2}-\mathrm{CON}\right), 2.27-2.20(\mathrm{~m}, 1 \mathrm{H}, \mathrm{CH}) \mathrm{ppm} .{ }^{13} \mathrm{C}-\mathrm{NMR}$ (Dioxane, $100 \mathrm{MHz}$ ) $\delta: 181.1$ (CO of acid), 163.2 (CO of amide), 132.5, 131.2 (2CH aromatic), 129.5 (2CH aromatic), 128.9, 59.2, 57.7, 32.3, $26.2 \mathrm{ppm}$. IR $(\mathrm{KBr}) \mathrm{cm}^{-1}: 3246(\mathrm{OH}$ of acid), 3075, 3053 ( $\mathrm{NH}$ two bands), 2983 ( $\mathrm{CH}$ aromatic), 2951 ( $\mathrm{CH}$ aliphatic), $1703(\mathrm{C}=\mathrm{O}$ of $\mathrm{COOH}), 1659(\mathrm{C}=\mathrm{O}$ amide), 1412 (OH bending in-plane), 1221, 1193 ( $\mathrm{SO}_{2}$ two bands), 696 (Ar-H), 631 (N-H bending with wagging). Anal. Calcd. for $300.34 \mathrm{C}_{12} \mathrm{H}_{16} \mathrm{~N}_{2} \mathrm{O}_{5} \mathrm{~S}$ : C, 47.99; H, 5.37; N, 9.33. Found: C, 48.03; H, 5.56; N, 9.41.

(10) 3-Phenyl-2-(phenylmethylsulfonamido)propanoic Acid, 10. L-amino acid is L-phenyl alanine; yield $=1.58 \mathrm{~g}(98.8 \%) ; \mathrm{mp}$ $158-159^{\circ} \mathrm{C}(\mathrm{dec}) ; R_{f}=0.84 .{ }^{1} \mathrm{H}-\mathrm{NMR}\left(\mathrm{D}_{2} \mathrm{O}, 400 \mathrm{MHz}\right) \delta$ : $7.46(\mathrm{~s}, 10 \mathrm{H}, 2 \times \mathrm{Ar}-\mathrm{H}), 4.34-4.31\left(\mathrm{dd}, J_{1}=5.60 \mathrm{~Hz}, J_{2}=\right.$ $\left.13.28 \mathrm{~Hz}, 1 \mathrm{H}, \mathrm{PhCH}_{2}-\mathrm{CH}-\mathrm{COOH}\right), 4.22$ (s, $2 \mathrm{H}, \mathrm{CH}_{2}-\mathrm{SO}_{2}$ ), $3.41-3.36\left(\mathrm{dd}, J_{1}=5.60 \mathrm{~Hz}, J_{2}=20.00 \mathrm{~Hz}, \mathrm{CHa}\right.$ of $\left.\mathrm{CH}_{2}-\mathrm{Ph}\right)$, $3.28-3.22\left(\mathrm{dd}, J_{1}=7.60 \mathrm{~Hz}, J_{2}=20 \mathrm{~Hz}, 1 \mathrm{H}, \mathrm{CHb}\right.$ of $\mathrm{CH}_{2}-$ $\mathrm{Ph}$ ) ppm. ${ }^{13} \mathrm{C}-\mathrm{NMR}$ (Dioxane, $100 \mathrm{MHz}$ ) $\delta: 172.3$ (CO of acid), 134.8, 132.6, 131.2 (2× $\mathrm{CH}$ aromatic), $130.3(2 \times$ $\mathrm{CH}$ aromatic $), 130.1(2 \times \mathrm{CH}$ aromatic $), 129.5(2 \times \mathrm{CH}$ aromatic), $128.9(2 \times \mathrm{CH}$ aromatic $), 57.7,55.0,36.4 \mathrm{ppm}$. IR $(\mathrm{KBr}) \mathrm{cm}^{-1}: 3441$ (OH of acid), $2974(\mathrm{NH}), 2822(\mathrm{CH}$ aromatic), 2776 (CH aliphatic), 1740 (C=O of $\mathrm{COOH}), 1609$ $(\mathrm{C}=\mathrm{C}), 1221,1171$ ( $\mathrm{SO}_{2}$ two bands), $702(\mathrm{Ar}-\mathrm{H}), 623(\mathrm{~N}-$ $\mathrm{H}$ bending). MS: in $m / z$ [rel. \%]: 270.1 [4\%], 212.1 [12\%], 180.1 [90\%], 179.1 [95\%], 178.1 [100\%], 165.1 [60\%], 122.0 [34\%], 121.0 [67\%], 64 [ $\left.\mathrm{SO}_{2}{ }^{+}, 70 \%\right]$. Anal. Calcd. for 319.38 $\mathrm{C}_{16} \mathrm{H}_{17} \mathrm{NO}_{4} \mathrm{~S}: \mathrm{C}, 60.17 ; \mathrm{H}, 5.37 ; \mathrm{N}, 4.39$. Found: C, 59.98; H, $5.21 ; \mathrm{N}, 4.42$.

(11) 3-(4-(Benzylsulfonyloxy)phenyl)-2-(phenylmethylsulfonamido)propanoic Acid, 11. L-amino acid is tyrosine; yield = $2.19 \mathrm{~g}(89.6 \%) ; \mathrm{mp} 195-196^{\circ} \mathrm{C} ; R_{f}=0.82 .{ }^{1} \mathrm{H}-\mathrm{NMR}\left(\mathrm{D}_{2} \mathrm{O}\right.$, $400 \mathrm{MHz}) \delta: 7.43(\mathrm{~s}, 10 \mathrm{H}, 2 \times \mathrm{Ar}-\mathrm{H}), 7.22-7.20(\mathrm{~d}, J=$ $8.4 \mathrm{~Hz}, 2 \mathrm{H}, \mathrm{OTs}-\mathrm{H}), 6.93-6.91$ (d, $J=8.4 \mathrm{~Hz}, 2 \mathrm{H}, \mathrm{OTs}-\mathrm{H})$, $4.31-4.28\left(\mathrm{dd}, J_{1}=5.60 \mathrm{~Hz}, J_{2}=13.08 \mathrm{~Hz}, 1 \mathrm{H}, \mathrm{PhCH}_{2}-\mathrm{CH}-\right.$ $\mathrm{COOH}), 4.19\left(\mathrm{~s}, 4 \mathrm{H}, 2 \times \mathrm{CH}_{2}-\mathrm{SO}_{2}\right), 3.31-3.26\left(\mathrm{dd}, J_{1}=\right.$ $5.60 \mathrm{~Hz}, J_{2}=20.00 \mathrm{~Hz}, \mathrm{CHa}$ of $\mathrm{CH}_{2}-\mathrm{Ph}$ ), 3.19-3.14 (dd, $J_{1}=$ $7.52 \mathrm{~Hz}, J_{2}=20 \mathrm{~Hz}, 1 \mathrm{H}, \mathrm{CHb}$ of $\left.\mathrm{CH}_{2}-\mathrm{Ph}\right)$ ppm. ${ }^{13} \mathrm{C}-\mathrm{NMR}$ (Dioxane, $100 \mathrm{MHz}$ ) $\delta: 172.8$ (CO of acid), 156.5, 140.9, 132.0, 131.8 (2CH aromatic), $131.3(2 \times \mathrm{CH}$ aromatic), 
$129.6(2 \times \mathrm{CH}$ aromatic $), 129.0(2 \times \mathrm{CH}$ aromatic $), 126.6$, $123.6(2 \times \mathrm{CH}$ aromatic $), 117.1(2 \times \mathrm{CH}$ aromatic $), 111.8$ $\left(2 \times \mathrm{CH}\right.$ aromatic), 58.4, 55.4, 36.1 ppm. IR $(\mathrm{KBr}) \mathrm{cm}^{-1}$ : 3435 (OH of acid), $3167(\mathrm{NH}), 3028$ (CH aromatic), 2949 ( $\mathrm{CH}$ aliphatic), 1724 ( $\mathrm{C}=\mathrm{O}$ of $\mathrm{COOH}), 1597(\mathrm{C}=\mathrm{C}), 1194$, 1148 ( $\mathrm{SO}_{2}$ two bands), 789 (S-OR ester), 694 (Ar-H), 631 (N-H bending). MS: in $m / z$ [rel. \%]: 180.1 [74\%], 179.1 [90\%], 178.1 [100\%], 165.1 [30\%], 122.0 [10\%], 91.1 $\left[\mathrm{PhCH}_{2}{ }^{+}, 47 \%\right], 64\left[\mathrm{SO}_{2}{ }^{+}, 24 \%\right]$. Anal. Calcd. for 489.57 $\mathrm{C}_{23} \mathrm{H}_{23} \mathrm{NO}_{7} \mathrm{~S}_{2}$ : C, 56.43; H, 4.76; N, 2.86. Found: C, 56.38; $\mathrm{H}, 4.79 ; \mathrm{N}, 2.69$.

4.2.2. General Procedure of N,N-Diethyl-2-(phenylmethylsulfonamido)propanamide,12-22. To a solution of $\alpha$-tolylsulfonamide derivatives $\mathbf{1 - 1 1}(2.96 \mathrm{mmol})$ in $\mathrm{H}_{2} \mathrm{O}(10 \mathrm{~mL})$ in a streaming flow of nitrogen gas was added oxalyl chloride ( $0.34 \mathrm{~mL}, 3.85 \mathrm{mmol}, 1.30$ eq.) via dropping pipette followed by carefully controlled addition of 1 drop of DMF. The resulting mixture was stirred at room temperature for $2 \mathrm{~h}$ to get crude acid chloride which was kept air-tighted prior to use. In a separate $250 \mathrm{~mL}$ three-necked round bottom flask, equipped with a magnetic stirring bar, was added $\mathrm{Na}_{2} \mathrm{CO}_{3}$ ( $0.628 \mathrm{~g}, 5.92 \mathrm{mmol}, 2$ equiv.) to $\mathrm{H}_{2} \mathrm{O}(10 \mathrm{~mL})$ followed by diethyl amine DEA $(0.4 \mathrm{~mL}, 3.85 \mathrm{mmol}, 1.3$ equiv. $)$ in a continuous stirring and cooled to $-15^{\circ} \mathrm{C}$. Then, earlier kept acid chloride was added in such a way to maintain the internal temperature of the reaction mixture at around $-10^{\circ} \mathrm{C}$. The reacting mixture was then stirred at $-10^{\circ} \mathrm{C}$ for $1 \mathrm{~h}$; at $0^{\circ} \mathrm{C}$ for $1 \mathrm{~h}$; finally at room temperature for $1 \mathrm{~h}$. The reaction was terminated, worked up by acidifying with $2 \mathrm{~N} \mathrm{HCl}$, and concentrated in a rotary evaporator. The clear solution obtained was freeze-dried to get crude solid product which was purified by column chromatography $\left(\mathrm{CHCl}_{3} / \mathrm{CH}_{3} \mathrm{OH}, 3: 1\right)$ to afford $N, N$-dimethylacetamide of $\alpha$-tolylsulfonamide derivatives 12-22.

(1) 1-(Benzylsulfonyl)-N,N-diethylpiperidine-2-carboxamide, 12. Yield $0.99 \mathrm{~g}(99.0 \%) ; \operatorname{mp} 210-211^{\circ} \mathrm{C} ; R_{f}=0.72 .{ }^{1} \mathrm{H}$ NMR $\left(\mathrm{D}_{2} \mathrm{O}, 400 \mathrm{MHz}\right) \delta: 7.54(\mathrm{~s}, 5 \mathrm{H}, \mathrm{Ar}-\mathrm{H}), 4.30(\mathrm{~s}, 2 \mathrm{H}$, $\mathrm{CH}_{2}-\mathrm{SO}_{2}$ ), 4.08-4.04 (dd, $J_{1}=3.52 \mathrm{~Hz}, J_{2}=20.00 \mathrm{~Hz}, 1 \mathrm{H}$, CHa of $\left.\mathrm{CH}_{2}-\mathrm{N}\right), 3.60-3.57(\mathrm{~m}, 1 \mathrm{H}, \mathrm{CH}-\mathrm{CON}), 3.21-3.16$ $\left(\mathrm{q}, J=7.20 \mathrm{~Hz}, 4 \mathrm{H}, 2 \times \mathrm{CH}_{2}-\mathrm{CH}_{3}\right), 2.42-2.38\left(\mathrm{dd}, J_{1}=\right.$ $3.32 \mathrm{~Hz}, J_{2}=20.00 \mathrm{~Hz}, 1 \mathrm{H}, \mathrm{CHb}$ of $\left.\mathrm{CH}_{2}-\mathrm{N}\right), 2.03-1.99(\mathrm{~m}$, $\left.2 \mathrm{H}, \mathrm{CH}_{2}\right), 1.86-1.73\left(\mathrm{~m}, 3 \mathrm{H}, \mathrm{CH} \& \mathrm{CH}_{2}\right), 1.40-1.37(\mathrm{t}, J$ $\left.=7.20 \mathrm{~Hz}, 6 \mathrm{H}, 2 \times \mathrm{CH}_{3}-\mathrm{CH}_{2}\right) \mathrm{ppm} .{ }^{13} \mathrm{C}-\mathrm{NMR}$ (Dioxane, $100 \mathrm{MHz}) \delta: 173.5(\mathrm{C}=\mathrm{O}), 132.9,131.1(2 \times \mathrm{CH}$ aromatic $)$, $129.8\left(2 \times \mathrm{CH}\right.$ aromatic), 128.6, 60.6, 57.8, 47.4, $43.4\left(\mathrm{CH}_{2}\right)$, 29.4, 24.2, 18.1, $11.4\left(\mathrm{CH}_{3}\right)$ ppm. IR $(\mathrm{KBr}) \mathrm{cm}^{-1}: 3028(\mathrm{CH}$ aromatic), 2951 ( $\mathrm{CH}$ aliphatic), $1720(\mathrm{C}=\mathrm{O}), 1593(\mathrm{C}=\mathrm{C})$, 1188, 1148 ( $\mathrm{SO}_{2}$ two bands), 696 (Ar-H). Anal. Calcd. for $\mathrm{C}_{17} \mathrm{H}_{26} \mathrm{~N}_{2} \mathrm{O}_{3} \mathrm{~S}$ (338.47): C, 60.33; H, 7.74; N, 8.28. Found: C, 60.29; H, 6.94; N, 7.98.

(2) 1-(Benzylsulfonyl)-N,N-diethylpyrrolidine-2-carboxamide, 13. Yield $0.94 \mathrm{~g}(97.9 \%)$; mp $185-187^{\circ} \mathrm{C} ; R_{f}=0.71 .{ }^{1} \mathrm{H}-$ $\operatorname{NMR}\left(\mathrm{D}_{2} \mathrm{O}, 400 \mathrm{MHz}\right) \delta: 7.53(\mathrm{~s}, 5 \mathrm{H}, \mathrm{Ar}-\mathrm{H}), 4.54-4.50(\mathrm{~m}$, $1 \mathrm{H}, \mathrm{CH}-\mathrm{CON}), 4.28\left(\mathrm{~s}, 2 \mathrm{H}, \mathrm{CH}_{2}-\mathrm{SO}_{2}\right), 3.56-3.54(\mathrm{~m}, 2 \mathrm{H}$, $\left.\mathrm{CH}_{2}-\mathrm{N}\right), 3.19-3.14\left(\mathrm{q}, J=7.28 \mathrm{~Hz}, 4 \mathrm{H}, 2 \times \mathrm{CH}_{2}-\mathrm{CH}_{3}\right)$,
2.54-2.51 (m, 1H, CH), 2.28-2.24 (m, 1H, CH), 2.16-2.14 $\left(\mathrm{m}, 2 \mathrm{H}, \mathrm{CH}_{2}\right), 1.39-1.35\left(\mathrm{t}, J=7.28 \mathrm{~Hz}, 6 \mathrm{H}, 2 \times \mathrm{CH}_{3}-\mathrm{CH}_{2}\right)$ ppm. ${ }^{13} \mathrm{C}-\mathrm{NMR}$ (Dioxane, $\left.100 \mathrm{MHz}\right) \delta: 173.2(\mathrm{C}=\mathrm{O}), 132.6$, $131.1(2 \times \mathrm{CH}$ aromatic), $129.4(2 \times \mathrm{CH}$ aromatic $), 128.8$, $60.8,57.7,47.1,43.3\left(2 \times \mathrm{CH}_{2}\right), 29.2,24.2,11.4\left(2 \times \mathrm{CH}_{3}\right)$ ppm. Anal. Calcd. for $\mathrm{C}_{16} \mathrm{H}_{24} \mathrm{~N}_{2} \mathrm{O}_{3} \mathrm{~S}$ (324.45): C, 59.23; $\mathrm{H}$, 7.46; N, 8.63. Found: C, 59.09; H, 7.46; N, 8.48.

(3) N,N-Diethyl-2-(phenylmethylsulfonamido)acetamide, 14. Yield $0.78 \mathrm{~g}(92.6 \%) ; \mathrm{mp} 213-215^{\circ} \mathrm{C} ; R_{f}=0.51 .{ }^{1} \mathrm{H}-\mathrm{NMR}$ $\left(\mathrm{D}_{2} \mathrm{O}, 400 \mathrm{MHz}\right) \delta: 7.53(\mathrm{~s}, 5 \mathrm{H}, \mathrm{Ar}-\mathrm{H}), 4.29\left(\mathrm{~s}, 2 \mathrm{H}, \mathrm{CH}_{2}-\right.$ $\mathrm{SO}_{2}$ ), 4.01 (s, $\left.2 \mathrm{H}, \mathrm{CH}_{2}-\mathrm{CON}\right), 3.20-3.14(\mathrm{q}, J=7.30 \mathrm{~Hz}, 4 \mathrm{H}$, $\left.2 \times \mathrm{CH}_{2}-\mathrm{CH}_{3}\right), 1.39-1.36\left(\mathrm{t}, J=7.30 \mathrm{~Hz}, 6 \mathrm{H}, 2 \times \mathrm{CH}_{3}-\mathrm{CH}_{2}\right)$ ppm. ${ }^{13} \mathrm{C}-\mathrm{NMR}$ (Dioxane, $\left.100 \mathrm{MHz}\right) \delta: 174.1(\mathrm{C}=\mathrm{O}), 133.4$, 131.6 $(2 \times \mathrm{CH}$ aromatic), $129.5(2 \times \mathrm{CH}$ aromatic $), 128.6$, 57.7, 49.8, $43.1\left(\mathrm{CH}_{2}\right), 11.4\left(\mathrm{CH}_{3}\right)$ ppm. IR $(\mathrm{KBr}) \mathrm{cm}^{-1}$ : 3217 (N-H), 3036 (CH aromatic), 2947 ( $\mathrm{CH}$ aliphatic), 1712 $(\mathrm{C}=\mathrm{O}), 1601$ (C=C), 1219, 1194, (SO 2 two bands), 694 (Ar$\mathrm{H})$. Anal. Calcd. for $\mathrm{C}_{13} \mathrm{H}_{20} \mathrm{~N}_{2} \mathrm{O}_{3} \mathrm{~S}$ (284.38): C, 54.91; H, 7.09; N, 9.85. Found: C, 55.13; H, 6.94; N, 10.08 .

(4) N,N-Diethyl-2-(phenylmethylsulfonamido)propanamide, 15. Yield $0.87 \mathrm{~g}(98.5 \%)$; mp $238-240^{\circ} \mathrm{C} ; R_{f}=0.56 .{ }^{1} \mathrm{H}-$ $\mathrm{NMR}\left(\mathrm{D}_{2} \mathrm{O}, 400 \mathrm{MHz}\right) \delta: 7.50(\mathrm{~s}, 5 \mathrm{H}, \mathrm{Ar}-\mathrm{H}), 4.26(\mathrm{~s}$, $\left.2 \mathrm{H}, \mathrm{CH}_{2}-\mathrm{SO}_{2}\right), 4.22-4.16\left(\mathrm{q}, J=7.28 \mathrm{~Hz}, 1 \mathrm{H}, \mathrm{CH}-\mathrm{CH}_{3}\right)$, $3.18-3.12\left(\mathrm{q}, J=7.32 \mathrm{~Hz}, 4 \mathrm{H}, 2 \times \mathrm{CH}_{2}-\mathrm{CH}_{3}\right), 1.65-1.63(\mathrm{~d}$, $\left.J=7.28 \mathrm{~Hz}, 3 \mathrm{H}, \mathrm{CH}_{3}-\mathrm{CH}\right), 1.37-1.33(\mathrm{t}, J=7.32 \mathrm{~Hz}, 6 \mathrm{H}, 2$ $\times \mathrm{CH}_{3}-\mathrm{CH}_{2}$ ) ppm. ${ }^{13} \mathrm{C}-\mathrm{NMR}$ (Dioxane, $\left.100 \mathrm{MHz}\right) \delta: 173.8$ $(\mathrm{C}=\mathrm{O}), 132.7,131.2(2 \times \mathrm{CH}$ aromatic $), 129.5(2 \times \mathrm{CH}$ aromatic), 128.9, 57.7, 49.8, $43.1\left(\mathrm{CH}_{2}\right), 16.2,11.4\left(\mathrm{CH}_{3}\right)$ ppm. IR (KBr) cm ${ }^{-1}: 3058(\mathrm{~N}-\mathrm{H}), 3036$ (CH aromatic), 2951 ( $\mathrm{CH}$ aliphatic), 1719 (C=O), 1601 ( $\mathrm{C}=\mathrm{C}), 1219,1196,1148$ ( $\mathrm{SO}_{2}$ two bands), 696 (Ar-H). Anal. Calcd. for $\mathrm{C}_{14} \mathrm{H}_{22} \mathrm{~N}_{2} \mathrm{O}_{3} \mathrm{~S}$ (298.41): C, 56.35; H, 7.43; N, 9.39. Found: C, 56.11; H, 7.33; N, 9.28.

(5) N,N-Diethyl-3-mercapto-2-(phenylmethylsulfonamido) propanamide, 16. Yield $0.87 \mathrm{~g}(89.0 \%) ; \mathrm{mp} 198-200^{\circ} \mathrm{C} ; R_{f}$ $=0.71 .{ }^{1} \mathrm{H}-\mathrm{NMR}\left(\mathrm{D}_{2} \mathrm{O}, 400 \mathrm{MHz}\right) \delta: 7.46(\mathrm{~s}, 5 \mathrm{H}, \mathrm{Ar}-\mathrm{H})$, $4.54-4.51\left(\mathrm{dd}, J_{1}=4.24 \mathrm{~Hz}, J_{2}=7.92 \mathrm{~Hz}, 1 \mathrm{H}, \mathrm{CH}_{2}-\mathrm{CH}-\right.$ $\mathrm{COOH}), 4.21\left(\mathrm{~s}, 2 \mathrm{H}, \mathrm{CH}_{2}-\mathrm{SO}_{2}\right), 3.57-3.52\left(\mathrm{dd}, J_{1}=4.24 \mathrm{~Hz}\right.$, $J_{2}=20.00 \mathrm{~Hz}, 1 \mathrm{H}, \mathrm{CHa}$ of $\left.\mathrm{CH}_{2}-\mathrm{CH}\right), 3.41-3.36\left(\mathrm{dd}, J_{1}=\right.$ $7.92 \mathrm{~Hz}, J_{2}=20.00 \mathrm{~Hz}, 1 \mathrm{H}, \mathrm{CHb}$ of $\left.\mathrm{CH}_{2}-\mathrm{CH}\right), 3.18-3.12(\mathrm{q}$, $\left.J=7.35 \mathrm{~Hz}, 4 \mathrm{H}, 2 \times \mathrm{CH}_{2}-\mathrm{CH}_{3}\right), 1.37-1.33(\mathrm{t}, J=7.35 \mathrm{~Hz}$, $6 \mathrm{H}, 2 \times \mathrm{CH}_{3}-\mathrm{CH}_{2}$ ) ppm. ${ }^{13} \mathrm{C}-\mathrm{NMR}$ (Dioxane, $100 \mathrm{MHz}$ ) $\delta: 173.5(\mathrm{C}=\mathrm{O}), 132.7,131.2(2 \times \mathrm{CH}$ aromatic $), 129.5(2$ $\times \mathrm{CH}$ aromatic), 128.9, 57.4, 49.5, $43.1\left(\mathrm{CH}_{2}\right), 35.5,11.4$ $\left(\mathrm{CH}_{3}\right)$ ppm. IR $(\mathrm{KBr}) \mathrm{cm}^{-1}: 2997(\mathrm{CH}$ aromatic), 2911 ( $\mathrm{CH}$ aliphatic), $1719(\mathrm{C}=\mathrm{O}), 1591(\mathrm{C}=\mathrm{C}), 1194,1144\left(\mathrm{SO}_{2}\right.$ two bands), 696 (Ar-H). Anal. Calcd. for $\mathrm{C}_{14} \mathrm{H}_{22} \mathrm{~N}_{2} \mathrm{O}_{3} \mathrm{~S}_{2}$ (330.47): C, 50.88; H, 6.71; N, 8.48. Found: C, 50.71; H, 6.99; N, 7.97 .

(6) N,N-Diethyl-4-(methylthio)-2-(phenylmethylsulfonamido) butanamide, 17. Yield $0.96 \mathrm{~g}(90.6 \%) ; \mathrm{mp} 170-172^{\circ} \mathrm{C} ; R_{f}=$ 0.65. ${ }^{1} \mathrm{H}-\mathrm{NMR}\left(\mathrm{D}_{2} \mathrm{O}, 400 \mathrm{MHz}\right) \delta: 7.49$ (s, 5H, Ar-H), 4.24 (s, $\left.2 \mathrm{H}, \mathrm{CH}_{2}-\mathrm{SO}_{2}\right), 4.23-4.20\left(\mathrm{t}, J=6.72 \mathrm{~Hz}, 1 \mathrm{H}, \mathrm{CH}-\mathrm{CH}_{2}\right)$, 3.16-3.10 (q, $\left.J=7.32 \mathrm{~Hz}, 4 \mathrm{H}, 2 \times \mathrm{CH}_{2}-\mathrm{CH}_{3}\right), 2.77-2.73(\mathrm{t}$, 
$\left.J=7.40 \mathrm{~Hz}, 2 \mathrm{H}, \mathrm{S}-\mathrm{CH}_{2}-\mathrm{CH}_{2}\right), 2.34-2.24\left(\mathrm{~m}, 2 \mathrm{H}, \mathrm{CH}-\mathrm{CH}_{2}-\right.$ $\mathrm{CH}_{2}-\mathrm{S}$ ), 2.19 (s, 3H, $\mathrm{CH}_{3}-\mathrm{S}$ ), 1.35-1.31 (t, J = 7.32 Hz, 6H, 2 $\times \mathrm{CH}_{3}-\mathrm{CH}_{2}$ ) ppm. ${ }^{13} \mathrm{C}-\mathrm{NMR}$ (Dioxane, $100 \mathrm{MHz}$ ) $\delta: 173.8$, 132.7, $131.2(2 \times \mathrm{CH}$ aromatic $), 129.5,(2 \times \mathrm{CH}$ aromatic $)$, 57.7, 49.8, 43.1 $\left(\mathrm{CH}_{2}\right), 30.8,29.5,16.2,11.4\left(\mathrm{CH}_{3}\right) \mathrm{ppm}$. IR $(\mathrm{KBr}) \mathrm{cm}^{-1}: 3028$ ( $\mathrm{CH}$ aromatic), 2945 ( $\mathrm{CH}$ aliphatic), $1722(\mathrm{C}=\mathrm{O}), 1620(\mathrm{C}=\mathrm{C}), 1200,1126$ ( $\mathrm{SO}_{2}$ two bands) 698 (Ar-H). Anal. Calcd. for $\mathrm{C}_{16} \mathrm{H}_{26} \mathrm{~N}_{2} \mathrm{O}_{3} \mathrm{~S}_{2}$ (358.53): C, 53.60; H, 7.31; N, 7.81. Found: C, 53.55; H, 7.22; N, 7.69.

(7) N,N-Diethyl-3-methyl-2-(phenylmethylsulfonamido)butanamide, 18. Yield $0.94 \mathrm{~g}(97.3 \%) ; \mathrm{mp} 226-230^{\circ} \mathrm{C} ; R_{f}=$ 0.69. ${ }^{1} \mathrm{H}-\mathrm{NMR}\left(\mathrm{D}_{2} \mathrm{O}, 400 \mathrm{MHz}\right) \delta: 7.50(\mathrm{~s}, 5 \mathrm{H}, \mathrm{Ar}-\mathrm{H})$, 4.25 (s, $2 \mathrm{H}, \mathrm{CH}_{2}-\mathrm{SO}_{2}$ ), 4.00-3.97 (d, $J=2.84 \mathrm{~Hz}, 1 \mathrm{H}, \mathrm{CH}-$ $\mathrm{CH}-\mathrm{CON}), 3.17-3.11\left(\mathrm{q}, \mathrm{J}=7.32 \mathrm{~Hz}, 4 \mathrm{H}, 2 \times \mathrm{CH}_{2}-\mathrm{CH}_{3}\right)$, 2.45-2.38 (m, 1H, CH), 1.36-1.32 (t, $J=7.32 \mathrm{~Hz}, 6 \mathrm{H}, 2$ $\left.\times \mathrm{CH}_{3}-\mathrm{CH}_{2}\right), 1.15-1.13\left(\mathrm{~d}, J=7.00 \mathrm{~Hz}, 3 \mathrm{H}, \mathrm{CH}_{3}-\mathrm{CH}\right)$, $1.12-1.10\left(\mathrm{~d}, J=7.00 \mathrm{~Hz}, 3 \mathrm{H}, \mathrm{CH}_{3}-\mathrm{CH}\right) \mathrm{ppm} .{ }^{13} \mathrm{C}-\mathrm{NMR}$ (Dioxane, $100 \mathrm{MHz}) \delta$ : $173.8(\mathrm{C}=\mathrm{O}), 132.7,131.2(2 \times \mathrm{CH}$ aromatic), $129.5(2 \times \mathrm{CH}$ aromatic $), 128.9,60.8,57.7,49.8$, $43.1\left(\mathrm{CH}_{2}\right), 16.2,15.5,11.4\left(\mathrm{CH}_{3}\right)$ ppm. IR $(\mathrm{KBr}) \mathrm{cm}^{-1}$ : 3053 ( $\mathrm{CH}$ aromatic), 2945 ( $\mathrm{CH}$ aliphatic), $1718(\mathrm{C}=\mathrm{O})$, 1611 (C=C), 1219, 1194 ( $\mathrm{SO}_{2}$ two bands), 696 (Ar-H). Anal. Calcd. for $\mathrm{C}_{16} \mathrm{H}_{26} \mathrm{~N}_{2} \mathrm{O}_{3} \mathrm{~S}$ (326.46): C, 58.87; H, 8.03; N, 8.58. Found: C, 59.01; H, 7.96; N, 8.61.

(8) N,N-Diethyl-3-hydroxy-2-(phenylmethylsulfonamido) butanamide, 19. Yield $0.78 \mathrm{~g}(80.2 \%) ; \mathrm{mp} 245^{\circ} \mathrm{C}$ (dec); $R_{f}$ $=0.53 .{ }^{1} \mathrm{H}-\mathrm{NMR}\left(\mathrm{D}_{2} \mathrm{O}, 400 \mathrm{MHz}\right) \delta: 7.55(\mathrm{~s}, 5 \mathrm{H}, \mathrm{Ar}-\mathrm{H})$, 4.54-4.52 (m, $\left.1 \mathrm{H}, \mathrm{CH}-\mathrm{CH}-\mathrm{CH}_{3}\right), 4.31$ (s, $\left.2 \mathrm{H}, \mathrm{CH}_{2}-\mathrm{SO}_{2}\right)$, 4.09-4.08 (d, $J=3.96 \mathrm{~Hz}, 1 \mathrm{H}, \mathrm{CH}-\mathrm{CH}-\mathrm{CON}$ ), 3.22-3.17 (q, $\left.J=7.32 \mathrm{~Hz}, 4 \mathrm{H}, 2 \times \mathrm{CH}_{2}-\mathrm{CH}_{3}\right), 2.85(\mathrm{~s}, 1 \mathrm{H}, \mathrm{OH}), 1.49-1.47$ $\left(\mathrm{d}, J=6.60 \mathrm{~Hz}, 3 \mathrm{H}, \mathrm{CH}_{3}-\mathrm{CH}\right), 1.41-1.37(\mathrm{t}, J=7.32 \mathrm{~Hz}$, $6 \mathrm{H}, 2 \times \mathrm{CH}_{3}-\mathrm{CH}_{2}$ ) ppm. ${ }^{13} \mathrm{C}-\mathrm{NMR}$ (Dioxane, $100 \mathrm{MHz}$ ) $\delta$ : $173.5(\mathrm{C}=\mathrm{O}), 132.9,131.3(2 \times \mathrm{CH}$ aromatic $), 129.5(2$ $\times \mathrm{CH}$ aromatic $), 128.9,61.2,58.1,49.5,42.5\left(\mathrm{CH}_{2}\right), 16.6$, $11.1\left(\mathrm{CH}_{3}\right)$ ppm. IR $(\mathrm{KBr}) \mathrm{cm}^{-1}: 3396(\mathrm{OH}), 3030(\mathrm{CH}$ aromatic), 2945 ( $\mathrm{CH}$ aliphatic), $1720(\mathrm{C}=\mathrm{O}), 1601(\mathrm{C}=\mathrm{C})$, 1221, 1194 ( $\mathrm{SO}_{2}$ two bands), 694 (Ar-H). Anal. Calcd. for $\mathrm{C}_{15} \mathrm{H}_{24} \mathrm{~N}_{2} \mathrm{O}_{4} \mathrm{~S}$ (328.43): C, 54.86; H, 7.37; N, 8.53. Found: C, 54.71; H, 7.26; N, 8.65.

(9) $N^{1}, N^{1}$-Diethyl-2-(phenylmethylsulfonamido)pentanediamide, 20. Yield $0.98 \mathrm{~g}(93.2 \%) ; \operatorname{mp~} 251-253^{\circ} \mathrm{C} ; R_{f}=0.58$. ${ }^{1} \mathrm{H}-\mathrm{NMR}\left(\mathrm{D}_{2} \mathrm{O}, 400 \mathrm{MHz}\right) \delta: 7.51$ (s, 5H, Ar-H), 4.52-4.48 $\left(\mathrm{dd}, J_{1}=5 \mathrm{~Hz}, J_{2}=14.32 \mathrm{~Hz}, 1 \mathrm{H}, \mathrm{NOC}-\mathrm{CH}-\mathrm{CH}_{2 a, b}\right), 4.26$ (s, $\left.2 \mathrm{H}, \mathrm{CH}_{2}-\mathrm{SO}_{2}\right), 3.17-3.12(\mathrm{q}, J=7.32 \mathrm{~Hz}, 4 \mathrm{H}, 2 \times$ $\left.\mathrm{CH}_{2}-\mathrm{CH}_{3}\right), 2.67-2.60\left(\mathrm{~m}, 1 \mathrm{H}, \mathrm{CH}_{a}\right.$ of $\left.\mathrm{CH}_{2}\right), 2.53-2.48(\mathrm{t}, J$ $\left.=8 \mathrm{~Hz}, 2 \mathrm{H}, \mathrm{CO}-\mathrm{CH}_{2}-\mathrm{CH}_{2}\right), 2.30-2.24\left(\mathrm{~m}, 1 \mathrm{H}, \mathrm{CH}_{b}\right.$ of $\left.\mathrm{CH}_{2}\right)$, $1.37-1.33\left(\mathrm{t}, J=7.32 \mathrm{~Hz}, 6 \mathrm{H}, 2 \times \mathrm{CH}_{3}-\mathrm{CH}_{2}\right) \mathrm{ppm} .{ }^{13} \mathrm{C}-\mathrm{NMR}$ (Dioxane, $100 \mathrm{MHz}) \delta$ : $182.7(\mathrm{C}=\mathrm{O}), 177.1(\mathrm{C}=\mathrm{O}), 132.6$, $131.2(2 \times \mathrm{CH}$ aromatic $), 129.5(2 \times \mathrm{CH}$ aromatic $), 128.9$, 57.7, 53.3, $43.1\left(\mathrm{CH}_{2}\right), 30.1,25.2,11.4\left(\mathrm{CH}_{3}\right)$ ppm. IR $(\mathrm{KBr})$ $\mathrm{cm}^{-1}$ : $3075(\mathrm{NH}), 3053$ (CH aromatic), 2951 (CH aliphatic), 1703 ( $\mathrm{C}=\mathrm{O}), 1659$ ( $\mathrm{C}=\mathrm{O}$ of $\mathrm{CON}), 1601$ ( $\mathrm{C}=\mathrm{C}), 1221,1193$ ( $\mathrm{SO}_{2}$ two bands), 696 (Ar-H). Anal. Calcd. for $\mathrm{C}_{16} \mathrm{H}_{25} \mathrm{~N}_{3} \mathrm{O}_{4} \mathrm{~S}$ (355.46): C, 54.06; H, 7.09; N, 11.82. Found: C, 53.95; H, $6.88 ; \mathrm{N}, 12.01$.
(10) N,N-Diethyl-3-phenyl-2-(phenylmethylsulfonamido) propanamide, 21. Yield $1.00 \mathrm{~g}(90.2 \%) ; \mathrm{mp} 227-229^{\circ} \mathrm{C}$; $R_{f}=0.70 .{ }^{1} \mathrm{H}-\mathrm{NMR}\left(\mathrm{D}_{2} \mathrm{O}, 400 \mathrm{MHz}\right) \delta: 7.49(\mathrm{~s}, 10 \mathrm{H}, 2$ $\times \mathrm{Ar}-\mathrm{H}), 4.39-4.36\left(\mathrm{dd}, J_{1}=5.60 \mathrm{~Hz}, J_{2}=7.60 \mathrm{~Hz}, 1 \mathrm{H}\right.$, NOC-CH-CH $2 a, b$ ), 4.24 (s, 2H, $\mathrm{CH}_{2}-\mathrm{SO}_{2}$ ), 3.44-3.39 (dd, $J_{1}=5.60 \mathrm{~Hz}, J_{2}=20.00 \mathrm{~Hz}, 1 \mathrm{H}, \mathrm{CH}_{a}$ of $\left.\mathrm{CH}_{2 a, b}\right), 3.31-3.26$ $\left(\mathrm{dd}, J_{1}=7.60 \mathrm{~Hz}, J_{2}=20.00 \mathrm{~Hz}, 1 \mathrm{H}, \mathrm{CH}_{b}\right.$ of $\left.\mathrm{CH}_{2 a, b}\right)$, 3.16-3.10 (q, $\left.J=7.32 \mathrm{~Hz}, 4 \mathrm{H}, 2 \times \mathrm{CH}_{2}-\mathrm{CH}_{3}\right), 2.78(\mathrm{~s}, 2 \mathrm{H}$, $\left.\mathrm{CH}_{2}\right), 1.35-1.31\left(\mathrm{t}, J=7.32 \mathrm{~Hz}, 6 \mathrm{H}, 2 \times \mathrm{CH}_{3}-\mathrm{CH}_{2}\right) \mathrm{ppm}$. ${ }^{13} \mathrm{C}-\mathrm{NMR}$ (Dioxane, $\left.100 \mathrm{MHz}\right) \delta: 172.4(\mathrm{C}=\mathrm{O}), 135.1,132.7$, $131.2(2 \times \mathrm{CH}$ aromatic), $130.3(2 \times \mathrm{CH}$ aromatic $), 130.1$ $(2 \times \mathrm{CH}$ aromatic $), 129.5(2 \times \mathrm{CH}$ aromatic $), 128.9(2 \times$ $\mathrm{CH}$ aromatic), 57.8, 55.2, $43.1\left(\mathrm{CH}_{2}\right), 36.5,11.5\left(\mathrm{CH}_{3}\right) \mathrm{ppm}$. IR (KBr) cm ${ }^{-1}$ : $2976(\mathrm{NH}), 2828(\mathrm{CH}$ aromatic), $2774(\mathrm{CH}$ aliphatic), $1736(\mathrm{C}=\mathrm{O}), 1620(\mathrm{C}=\mathrm{C}), 1206,1153,1148\left(\mathrm{SO}_{2}\right.$ two bands), 698 (Ar-H). Anal. Calcd. for $\mathrm{C}_{20} \mathrm{H}_{26} \mathrm{~N}_{2} \mathrm{O}_{3} \mathrm{~S}$ (374.51): C, 64.14; H, 7.00; N, 7.48. Found: C, 64.00; H, 6.84; N, 7.29.

(11) 4-(3-(Diethylamino)-3-oxo-2-(phenylmethylsulfonamido) $\mathrm{pr}$ opyl)phenylphenylmethanesulfonate, 22. Yield $1.44 \mathrm{~g}(89.3 \%)$; $\mathrm{mp} 265^{\circ} \mathrm{C}(\mathrm{dec}) ; R_{f}=0.69 .{ }^{1} \mathrm{H}-\mathrm{NMR}\left(\mathrm{D}_{2} \mathrm{O}, 400 \mathrm{MHz}\right) \delta$ : $7.50(\mathrm{~s}, 10 \mathrm{H}, 2 \times \mathrm{Ar}-\mathrm{H}), 7.29-7.27(\mathrm{~d}, J=8.00 \mathrm{~Hz}, 2 \mathrm{H}, \mathrm{Ar}-$ $\mathrm{H}), 6.99-6.97$ (d, $J=8.00 \mathrm{~Hz}, 2 \mathrm{H}, \mathrm{Ar}-\mathrm{H}), 4.37-4.33$ (dd, $J_{1}$ $\left.=5.60 \mathrm{~Hz}, J_{2}=7.60 \mathrm{~Hz}, 1 \mathrm{H}, \mathrm{NOC}-\mathrm{CH}-\mathrm{CH}_{2 a, b}\right), 4.26(\mathrm{~s}, 2 \mathrm{H}$, $\left.\mathrm{CH}_{2}-\mathrm{SO}_{2}\right), 3.37-3.32\left(\mathrm{dd}, J_{1}=5.60 \mathrm{~Hz}, J_{2}=20.00 \mathrm{~Hz}, 1 \mathrm{H}\right.$, $\mathrm{CH}_{a}$ of $\left.\mathrm{CH}_{2 a, b}\right), 3.26-3.21\left(\mathrm{dd}, J_{1}=7.60 \mathrm{~Hz}, J_{2}=20.00 \mathrm{~Hz}\right.$, $1 \mathrm{H}, \mathrm{CH}_{b}$ of $\left.\mathrm{CH}_{2 a, b}\right), 3.17-3.12\left(\mathrm{q}, J=7.32 \mathrm{~Hz}, 4 \mathrm{H}, 2 \times \mathrm{CH}_{2}-\right.$ $\left.\mathrm{CH}_{3}\right), 1.37-1.33\left(\mathrm{t}, J=7.32 \mathrm{~Hz}, 6 \mathrm{H}, 2 \times \mathrm{CH}_{3}-\mathrm{CH}_{2}\right) \mathrm{ppm}$. ${ }^{13} \mathrm{C}-\mathrm{NMR}$ (Dioxane, $\left.100 \mathrm{MHz}\right) \delta: 172.4(\mathrm{C}=\mathrm{O}), 155.9,141.3$, $131.8(2 \times \mathrm{CH}$ aromatic $), 131.3(2 \times \mathrm{CH}$ aromatic $), 129.6$ $(2 \times \mathrm{CH}$ aromatic $), 129.0(2 \times \mathrm{CH}$ aromatic $), 126.6,123.0$ $(2 \times \mathrm{CH}$ aromatic $), 116.9(2 \times \mathrm{CH}$ aromatic $), 111.6(2 \times$ $\mathrm{CH}$ aromatic $), 57.8,55.2,43.2\left(\mathrm{CH}_{2}\right), 35.6,11.6\left(\mathrm{CH}_{3}\right) \mathrm{ppm}$. IR $(\mathrm{KBr}) \mathrm{cm}^{-1}: 3058(\mathrm{NH}), 3036(\mathrm{CH}$ aromatic), $2951(\mathrm{CH}$ aliphatic), $1719(\mathrm{C}=\mathrm{O}), 1601(\mathrm{C}=\mathrm{C}), 1219,1196,1148\left(\mathrm{SO}_{2}\right.$ two bands), 696 (Ar-H). Anal. Calcd. for $\mathrm{C}_{27} \mathrm{H}_{32} \mathrm{~N}_{2} \mathrm{O}_{6} \mathrm{~S}_{2}$ (544.69): C, 59.54; H, 5.92; N, 5.14. Found: C, 59.43; H, 5.99; N, 4.98 .

4.3. Antibacterial Activity Assays. The antimicrobial properties of the sulfonamides were investigated in the form of the general sensitivity testing and minimum inhibitory concentration (MIC) with respect to freshly cultured targeted organisms. The two organisms of interest in this present study are one gram positive (Staphylococcus aureus ATCC 6538) and one gram negative (Escherichia coli ATCC 25922) organisms which are associated with the gastrointestinal tract damage in man and animal.

4.3.1. Preparation of the Inoculum. The standard strains of $S$. aureus and E. coli used were obtained from Test Center of Antimicrobial Materials, TIPC, Beijing. No clinically isolated organism was used based on inavailability of such as at the time of this study. The strains were propagated on nutrient agar plates and maintained on the plate at $4^{\circ} \mathrm{C}$. The isolates were subcultured in nutrient broth at $37^{\circ} \mathrm{C}$ for $8 \mathrm{~h}$ prior to antibacterial testing. 
4.3.2. Antibacterial Sensitivity Testing of the Synthesized Compounds. Agar well diffusion technique as described by Adeniyi and coworkers was used to determine the antibacterial activity of the synthesized compounds [66]. Sensitivity test agar plates were seeded with $0.1 \mathrm{~mL}$ of an overnight culture of each bacterial strain (equivalent to $10^{7}-10^{8}$ $\mathrm{CFU} \mathrm{mL} \mathrm{m}^{-1}$ ). The seeded plates were allowed to set and a standard cork borer of $8 \mathrm{~mm}$ diameter was used to cut uniform wells on the surface of the agar. The wells were then filled with $0.3 \mathrm{~mL}$ of each sulfonamide solution in an appropriate solvent at a concentration of $1000 \mu \mathrm{g} / \mathrm{mL}$ ( $0.02 \mathrm{~g}$ of sulfonamide dissolved in $20 \mathrm{~mL}$ distilled water). All the plates were incubated at $37^{\circ} \mathrm{C}$ for $24 \mathrm{~h}$. The assay was conducted at regular intervals of $24 \mathrm{~h}$ until a marked decline in the potency of the sulfonamide solution to inhibit the growth of the test organisms was noticed. Zones of clearance round each well means inhibition and the diameter of such zones were measured. The procedure was repeated for the streptomycin (standard).

\subsubsection{Determination of Minimum Inhibitory Concentration} (MIC). Agar well dilution method as described by Russell and Furr was used to determine the minimum inhibitory concentration (MIC) of the sulfonamides and streptomycin [69]. Different dilutions of the sulfonamides were prepared first at $\leq 100 \mu \mathrm{g} / \mathrm{mL}$ to give final concentrations in the range of $100,50,25,12.5,6.25$, and $1.8 \mu \mathrm{g} / \mathrm{mL}$. The different dilutions of sulfonamide derivatives that could not inhibit the microbial growth at $\leq 100 \mu \mathrm{g} / \mathrm{mL}$ were later prepared at $\leq 1000 \mu \mathrm{g} / \mathrm{mL}$ to give final concentrations in the range of $1000,500,250,125$, and $62.5 \mu \mathrm{g} / \mathrm{mL}$. Two milliliter $(2 \mathrm{~mL})$ of each dilution was mixed with $18 \mathrm{~mL}$ of Mueller Hinton agar (MHA, Difco, France) and poured into Petri dishes and allowed to set. The agar was streaked with an overnight broth culture of the bacterial strains and incubated overnight. The plates were then examined for the presence or absence of growth. The minimum concentration that completely inhibited macroscopic growth was regarded as the minimum inhibitory concentration of the respective sulfonamide. The procedure was repeated for streptomycin (standard). Selectivity index (SI) is the ratio of the zone of the inhibition of compound to that of the streptomycin.

\section{Conflict of Interests}

The authors hereby declare that there is no conflict of interest as regard this paper.

\section{Acknowledgments}

O. O. Ajani is grateful to the Academy of Science for the Developing World (TWAS) and Chinese Academy of Sciences (CAS) for the sponsorship of this work. This present research work was birthed from the 2009 TWAS-CAS PG Fellowship Award which was jointly funded by both TWAS and CAS.

\section{References}

[1] M. A. Bhat, M. Imran, S. A. Khan, and N. Siddiqui, "Biological activities of sulfonamides," Indian Journal of Pharmaceutical Sciences, vol. 67, no. 2, pp. 151-159, 2005.

[2] C. T. Supuran, A. Casini, A. Mastrolorenzo, and A. Scozzafava, "COX-2 selective inhibitors, carbonic anhydrase inhibition and anticancer properties of sulfonamides belonging to this class of pharmacological agents," Mini-Reviews in Medicinal Chemistry, vol. 4, no. 6, pp. 625-632, 2004.

[3] A. K. Gadad, C. S. Mahajanshetti, S. Nimbalkar, and A. Raichurkar, "Synthesis and antibacterial activity of some 5-guanylhydrazone/thiocyanato-6-arylimidazo[2,1-b]1,3,4-thiadiazole-2-sulfon amide derivatives," European Journal of Medicinal Chemistry, vol. 35, no. 9, pp. 853-857, 2000.

[4] H. Eshghi, M. Rahimizadeh, M. Zokaei et al., "Synthesis and antimicrobial activity of some new macrocyclic bissulfonamide and disulphides," European Journal of Chemistry, vol. 2, no. 1, pp. 47-50, 2011.

[5] D. W. Hopper, M. D. Vera, D. How et al., "Synthesis and biological evaluation of ((4-keto)-phenoxy)methyl biphenyl4-sulfonamides: a class of potent aggrecanase-1 inhibitors," Bioorganic and Medicinal Chemistry Letters, vol. 19, no. 9, pp. 2487-2491, 2009.

[6] C. Juli, M. Sippel, J. Jäger et al., "Pipecolic acid derivatives as small-molecule inhibitors of the legionella MIP protein," Journal of Medicinal Chemistry, vol. 54, no. 1, pp. 277-283, 2011.

[7] A. Scozzafava and C. T. Supuran, "Protease inhibitorspart 5. Alkyl/arylsulfonyl- and arylsulfonylureido-/arylureidoglycine hydroxamate inhibitors of Clostridium histolyticum collagenase," European Journal of Medicinal Chemistry, vol. 35, no. 3, pp. 299-307, 2000.

[8] J. Schröder, A. Henke, H. Wenzel et al., "Structure-based design and synthesis of potent matrix metalloproteinase inhibitors derived from a $6 \mathrm{H}$-1,3,4-thiadiazine scaffold," Journal of Medicinal Chemistry, vol. 44, no. 20, pp. 3231-3243, 2001.

[9] D. F. Burdi, S. Chi, K. Mattia et al., "Small molecule antagonists of the CC chemokine receptor 4 (CCR4)," Bioorganic and Medicinal Chemistry Letters, vol. 17, no. 11, pp. 3141-3145, 2007.

[10] G. A. Domagk, "A contribution to chemotherapy of bacterial infections," Clinical Infectious Diseases, vol. 8, no. 1, pp. 163166, 1986.

[11] D. Vicente and E. Pérez-Trallero, "Tetracyclines, sulfonamides, and metronidazole," Enfermedades Infecciosas y Microbiología Clínica, vol. 28, no. 2, pp. 122-130, 2010.

[12] Z. Chen, W. Xu, K. Liu et al., "Synthesis and antiviral activity of 5-(4-chlorophenyl)-1,3,4-thiadiazole sulfonamides," Molecules, vol. 15, no. 12, pp. 9046-9056, 2010.

[13] A. J. McCarroll, T. D. Bradshaw, A. D. Westwell, C. S. Matthews, and M. F. G. Stevens, "Quinols as novel therapeutic agents. 7. ${ }^{1}$ Synthesis of antitumor 4-[1-(arylsulfonyl-1Hindol-2-yl)]-4-hydroxycyclohexa-2,5-dien-1-ones by Sonogashira reactions," Journal of Medicinal Chemistry, vol. 50, no. 7, pp. 1707-1710, 2007.

[14] B. L. Wilkinson, L. F. Bornaghi, T. A. Houston et al., "Carbonic anhydrase inhibitors: Inhibition of isozymes I, II, and IX with triazole-linked O-glycosides of benzene sulfonamides," Journal of Medicinal Chemistry, vol. 50, no. 7, pp. 1651-1657, 2007.

[15] A. Ali, G. S. K. K. Reddy, H. Cao et al., "Discovery of HIV1 protease inhibitors with picomolar affinities incorporating $\mathrm{N}$-aryl-oxazolidinone-5-carboxamides as novel P2 ligands," 
Journal of Medicinal Chemistry, vol. 49, no. 25, pp. 7342-7356, 2006.

[16] H. Kourlas and S. Morey, "Sulfonamide allergies and possible cross-reactivity," Journal of Pharmacy Practice, vol. 20, no. 5, pp. 399-402, 2007.

[17] M. Gurrath, "Peptide-binding G protein-coupled receptors: new opportunities for drug design," Current Medicinal Chemistry, vol. 8, no. 13, pp. 1605-1648, 2001.

[18] H. T. Lai and J. H. Hou, "Light and microbial effects on the transformation of four sulfonamides in eel pond water and sediment," Aquaculture, vol. 283, no. 1-4, pp. 50-55, 2008.

[19] M. J. O. 'Neil, P. E. Heckelman, C. B. Koch, and K. J. Roman, Eds., The Merck Index, Merck, Whitehouse Station, NJ, USA, 14th edition, 2006.

[20] N. Anand and W. A. Remers, Burger's Medicinal Chemistry, Drug Discovery and Development, John Wiley \& Sons, New York, NY, USA, 2010.

[21] D. E. Golan, "Dihydrofolate reductase inhibitors," in Principle of Pharmacology: The Pathophysiologic Basis of Drug Therapy, D. E. Golan, A. H. Tashjian, E. J. Armstrong, and A. W. Armstrong, Eds., pp. 577-580, Lippincott Williams \& Wilkins, Philadelphia, Pa, USA, 2nd edition, 2008.

[22] P. Purushottamachar, A. Khandelwal, T. S. Vasaitis, R. D. Bruno, L. K. Gediya, and V. C. O. Njar, "Potent anti-prostate cancer agents derived from a novel androgen receptor downregulating agent," Bioorganic and Medicinal Chemistry, vol. 16, no. 7, pp. 3519-3529, 2008.

[23] B. R. Stranix, J. F. Lavallée, G. Sévigny et al., "Lysine sulfonamides as novel HIV-protease inhibitors: $\mathrm{N} \varepsilon$-acyl aromatic $\alpha$ amino acids," Bioorganic and Medicinal Chemistry Letters, vol. 16, no. 13, pp. 3459-3462, 2006.

[24] W. G. Harter, H. Albrect, K. Brady et al., "The design and synthesis of sulfonamides as caspase-1 inhibitors," Bioorganic and Medicinal Chemistry Letters, vol. 14, no. 3, pp. 809-812, 2004.

[25] N. S. Reddy, M. R. Mallireddigari, S. Cosenza et al., "Synthesis of new coumarin 3-(N-aryl) sulfonamides and their anticancer activity," Bioorganic and Medicinal Chemistry Letters, vol. 14, no. 15, pp. 4093-4097, 2004.

[26] F. Abbate, A. Casini, T. Owa, A. Scozzafava, and C. T. Supuran, "Carbonic anhydrase inhibitors: E7070, a sulfonamide anticancer agent, potently inhibits cytosolic isozymes I and II, and transmembrane, tumor-associated isozyme IX," Bioorganic and Medicinal Chemistry Letters, vol. 14, no. 1, pp. 217-223, 2004.

[27] K. Na and Y. H. Bae, "Self-assembled hydrogel nanoparticles responsive to tumor extracellular $\mathrm{pH}$ from pullulan derivative/sulfonamide conjugate: characterization, aggregation, and adriamycin release in vitro," Pharmaceutical Research, vol. 19, no. 5, pp. 681-688, 2002.

[28] K. Fukuoka, J. Usuda, Y. Iwamoto et al., "Mechanisms of action of the novel sulfonamide anticancer agent E7070 on cell cycle progression in human non-small cell lung cancer cells," Investigational New Drugs, vol. 19, no. 3, pp. 219-227, 2001.

[29] A. Weber, A. Casini, A. Heine et al., "Unexpected nanomolar inhibition of carbonic anhydrase by COX-2-selective celecoxib: new pharmacological opportunities due to related binding site recognition," Journal of Medicinal Chemistry, vol. 47, no. 3, pp. 550-557, 2004.

[30] A. G. Habeeb, P. N. P. Rao, and E. E. Knaus, "Design and synthesis of celecoxib and rofecoxib analogues as selective cyclooxygenase-2 (COX-2) inhibitors: replacement of sulfonamide and methylsulfonyl pharmacophores by an azido bioisostere," Journal of Medicinal Chemistry, vol. 44, no. 18, pp. 3039-3042, 2001.

[31] A. L. Blobaum and L. J. Marnett, "Structural and functional basis of cyclooxygenase inhibition," Journal of Medicinal Chemistry, vol. 50, no. 7, pp. 1425-1441, 2007.

[32] I. R. Ezabadi, C. Camoutsis, P. Zoumpoulakis et al., "Sulfonamide-1,2,4-triazole derivatives as antifungal and antibacterial agents: synthesis, biological evaluation, lipophilicity, and conformational studies," Bioorganic and Medicinal Chemistry, vol. 16, no. 3, pp. 1150-1161, 2008.

[33] P. E. Sum, A. T. Ross, P. J. Petersen, and R. T. Testa, "Synthesis and antibacterial activity of 9-substituted minocycline derivatives," Bioorganic and Medicinal Chemistry Letters, vol. 16, no. 2, pp. 400-403, 2006.

[34] S. Joshi, N. Khosla, D. Khare, and R. Sharda, "Synthesis and in vitro study of novel Mannich bases as antibacterial agents," Bioorganic and Medicinal Chemistry Letters, vol. 15, no. 1, pp. 221-226, 2005.

[35] M. Padmanilayam, B. Scorneaux, Y. Dong et al., "Antimalarial activity of $\mathrm{N}$-alkyl amine, carboxamide, sulfonamide, and urea derivatives of a dispiro-1,2,4-trioxolane piperidine," Bioorganic and Medicinal Chemistry Letters, vol. 16, no. 21, pp. 5542-5545, 2006.

[36] J. N. Domínguez, C. León, J. Rodrigues, N. G. De Domínguez, J. Gut, and P. J. Rosenthal, "Synthesis and antimalarial activity of sulfonamide chalcone derivatives," Farmaco, vol. 60, no. 4, pp. 307-311, 2005.

[37] G. Bouchain, S. Leit, S. Frechette et al., "Development of potential antitumor agents. Synthesis and biological evaluation of a new set of sulfonamide derivatives as histone deacetylase inhibitors," Journal of Medicinal Chemistry, vol. 46, no. 5, pp. 820-830, 2003.

[38] A. Yokoi, J. Kuromitsu, T. Kawai et al., "Profiling novel sulfonamide antitumor agents with cell-based phenotypic screens and array-based gene expression analysis," Molecular Cancer Therapeutics, vol. 1, no. 4, pp. 275-286, 2002.

[39] M. Banerjee, A. Poddar, G. Mitra, A. Surolia, T. Owa, and B. Bhattacharyya, "Sulfonamide drugs binding to the colchicine site of tubulin: thermodynamic analysis of the drug-tubulin interactions by isothermal titration calorimetry," Journal of Medicinal Chemistry, vol. 48, no. 2, pp. 547-555, 2005.

[40] T. Inaba, K. Tanaka, R. Takeno, H. Nagaki, C. Yoshida, and S. Takano, "Synthesis and antiinflammatory activity of 7methanesulfonylamino-6-phenoxychromones. Antiarthritic effect of the 3-formylamino compound (T-614) in chronic inflammatory disease models," Chemical and Pharmaceutical Bulletin, vol. 48, no. 1, pp. 131-139, 2000.

[41] J. J. Talley, S. R. Bertenshaw, D. L. Brown et al., " $N-[[(5-$ methyl-3-phenylisoxazol-4-yl)phenyl]sulfonyl]propanamide, sodium salt, parecoxib sodium: a potent and selective inhibitor of COX-2 for parenteral administration," Journal of Medicinal Chemistry, vol. 43, no. 9, pp. 1661-1663, 2000.

[42] S. M. Sondhi, M. Johar, N. Singhal, S. G. Dastidar, R. Shukla, and R. Raghubir, "Synthesis and anticancer, antiinflammatory, and analgesic activity evaluation of some sulfa drug and acridine derivatives," Monatshefte fur Chemie, vol. 131, no. 5, pp. 511-520, 2000.

[43] K. Chibale, H. Haupt, H. Kendrick et al., "Antiprotozoal and cytotoxicity evaluation of sulfonamide and urea analogues of quinacrine," Bioorganic and Medicinal Chemistry Letters, vol. 11, no. 19, pp. 2655-2657, 2001.

[44] C. Brisbare-Roch, J. Dingemanse, R. Koberstein et al., "Promotion of sleep by targeting orexin system in rats, dogs and humans," Nature Medicine, vol. 13, no. 2, pp. 150-155, 2007. 
[45] M. G. Lee, O. K. Hassani, and B. E. Jones, "Discharge of identified orexin/hypocretin neurons across the sleep-waking cycle," Journal of Neuroscience, vol. 25, no. 28, pp. 6716-6720, 2005.

[46] I. V. Estabrooke, M. T. McCarthy, E. Ko et al., "Fos expression in orexin neurons varies with behavioral state," Journal of Neuroscience, vol. 21, no. 5, pp. 1656-1662, 2001.

[47] J. Bhaumik, R. Weissleder, and J. R. McCarthy, "Synthesis and photophysical properties of sulfonamidophenyl porphyrins as models for activatable photosensitizers," Journal of Organic Chemistry, vol. 74, no. 16, pp. 5894-5901, 2009.

[48] M. A. Gonzalez, D. B. Gorman, C. T. Hamilton, and G. A. Roth, "Process development herbicide pyroxsulam," Organic Process Research and Development, vol. 12, no. 2, pp. 301-303, 2008.

[49] T. Arslan, F. Kandemirli, E. E. Ebenso, I. Love, and H. Alemu, "Quantum chemical studies on the corrosion inhibition of some sulphonamides on mild steel in acidic medium," Corrosion Science, vol. 51, no. 1, pp. 35-47, 2009.

[50] M. M. El-Naggar, "Corrosion inhibition of mild steel in acidic medium by some sulfa drugs compounds," Corrosion Science, vol. 49, no. 5, pp. 2226-2236, 2007.

[51] N. B. Dyatkina, C. D. Roberts, J. D. Keicher et al., "Minor groove DNA binders as antimicrobial agents. 1. Pyrrole tetraamides are potent antibacterials against vancomycin resistant Enteroccoci and methicillin resistant Staphylococcus aureus," Journal of Medicinal Chemistry, vol. 45, no. 4, pp. 805$817,2002$.

[52] G. W. Kaatz, F. McAleese, and S. M. Seo, "Multidrug resistance in Staphylococcus aureus due to overexpression of a novel multidrug and toxin extrusion (MATE) transport protein," Antimicrobial Agents and Chemotherapy, vol. 49, no. 5, pp. 1857-1864, 2005.

[53] N. Nayak, T. C. Nag, G. Satpathy, and S. B. Ray, "Ultrastructural analysis of slime positive \& slime negative Staphylococcus epidermidis isolates in infectious keratitis," Indian Journal of Medical Research, vol. 125, no. 6, pp. 767-771, 2007.

[54] A. Masunari and L. C. Tavares, "A new class of nifuroxazide analogues: synthesis of 5-nitrothiophene derivatives with antimicrobial activity against multidrug-resistant Staphylococcus aureus," Bioorganic and Medicinal Chemistry, vol. 15, no. 12, pp. 4229-4236, 2007.

[55] K. Nishino, S. Yamasaki, M. Hayashi-Nishino, and A. Yamaguchi, "Effect of NlpE overproduction on multidrug resistance in Escherichia coli," Antimicrobial Agents and Chemotherapy, vol. 54, no. 5, pp. 2239-2243, 2010.

[56] K. E. Jones, N. G. Patel, M. A. Levy et al., "Global trends in emerging infectious diseases," Nature, vol. 451, no. 7181, pp. 990-993, 2008.

[57] P. K. Anderson, A. A. Cunningham, N. G. Patel, F. J. Morales, P. R. Epstein, and P. Daszak, "Emerging infectious diseases of plants: pathogen pollution, climate change and agrotechnology drivers," Trends in Ecology and Evolution, vol. 19, no. 10, pp. 535-544, 2004.

[58] O. C. Nwinyi, S. N. Chinedu, O. O. Ajani, C. O. Ikpo, and K. O. Ogunniran, "Antibacterial effects of extracts of Ocimum gratissimum and piper guineense on Escherichia coli and Staphylococcus aureus," African Journal of Food Science, vol. 3, no. 3, pp. 77-81, 2009.

[59] D. A. A. Mossel and P. van Netten, "Staphylococcus aureus and related Staphylococci in foods: ecology, proliferation, toxinogenesis, control and monitoring," Journal of Applied Bacteriology Symposium Supplement, vol. 69, no. 19, pp. 123 146,1990
[60] O. C. Nwinyi, N. S. Chinedu, and O. O. Ajani, "Evaluation of antibacterial activity of Pisidium guajava and Gongronema latifolium," Journal of Medicinal Plant Research, vol. 2, no. 8, pp. 189-192, 2008.

[61] G. E. Dolores and J. G. Doyle, "Escherichia coli in diarrhea disease," Annals of Internal Medicine, vol. 247, no. 1, pp. 8190, 2001.

[62] O. O. Ajani, C. A. Obafemi, O. C. Nwinyi, and D. A. Akinpelu, "Microwave assisted synthesis and antimicrobial activity of 2-quinoxalinone-3-hydrazone derivatives," Bioorganic and Medicinal Chemistry, vol. 18, no. 1, pp. 214-221, 2010.

[63] O. O. Ajani and O. C. Nwinyi, "Microwave-assisted synthesis and evaluation of antimicrobial activity of 3-\{3-(s-aryl and $s$-heteroaromatic)acryloyl $\}-2 H$-chromen-2-one derivatives," Journal of Heterocyclic Chemistry, vol. 47, no. 1, pp. 179$187,2010$.

[64] W. Zhang, Y. Qin, S. Zhang, and M. Luo, "Synthesis of novel $\mathrm{N}$-heterocyclic carbene-Rh complexes derived from L-proline and their catalysis in the addition of arylboronic acids to aldehydes," Arkivoc, vol. 2005, no. 14, pp. 39-48, 2005.

[65] J. T. Kuethe and G. L. Beutner, "Synthesis of 2-arylindole4-carboxylic amides: [2(4-fluorophenyl)-1H-indol-4-yl]-1pyrrolidinylmethanone," Organic Syntheses, vol. 86, no. 1, pp. 92-104, 2009.

[66] B. A. Adeniyi, H. A. Odelola, and B. A. Oso, "Antimicrobial potentials of Diospyros mespiliformis (Ebenaceae)," African Journal of Medical and Medicinal Science, vol. 25, no. 3, pp. 221-224, 1996.

[67] D. Voet and J. D. Voet, "The genetic code," in Biochemistry, pp. 1341-1342, John Wiley \& Sons, New York, NY, USA, 3rd edition, 2004.

[68] O. O. Ajani and O. C. Nwinyi, "Synthesis and evaluation of antimicrobial activity of phenyl and furan-2yl $[1,2,4]$ triazolo[4,3-a]quinoxalin-4 $(5 H)$-one and their hydrazone precursors," Canadian Journal of Pure and Applied Sciences, vol. 3, no. 3, pp. 983-992, 2009.

[69] A. D. Russell and J. R. Furr, "The antibacterial activity of a new chloroxylenol preparation containing ethylenediamine tetraacetic acid," Journal of Applied Bacteriology, vol. 43, no. 2, pp. 253-260, 1977.

[70] C. R. Andrighetti-Fröhner, K. N. De Oliveira, D. GasparSilva et al., "Synthesis, biological evaluation and SAR of sulfonamide 4-methoxychalcone derivatives with potential antileishmanial activity," European Journal of Medicinal Chemistry, vol. 44, no. 2, pp. 755-763, 2009.

[71] H. Aissaoui, R. Koberstein, C. Zumbrunn et al., "N-glycinesulfonamides as potent dual orexin 1/orexin 2 receptor antagonists," Bioorganic and Medicinal Chemistry Letters, vol. 18, no. 21, pp. 5729-5733, 2008.

[72] E. Pérez-Trallero and L. Iglesias, "Tetracyclines, sulfonamides and metronidazole," Enfermedades Infecciosas y Microbiología Clínica, vol. 21, no. 9, pp. 520-533, 2003.

[73] I. Levin, M. Mevarech, and B. A. Palfey, "Characterization of a novel bifunctional dihydropteroate synthase/dihydropteroate reductase enzyme from Helicobacter pylori," Journal of Bacteriology, vol. 189, no. 11, pp. 4062-4069, 2007. 


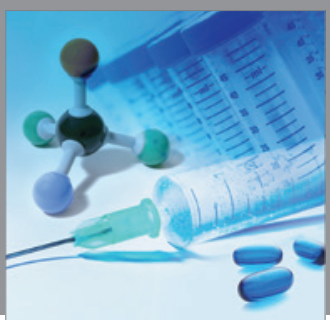

International Journal of

Medicinal Chemistry

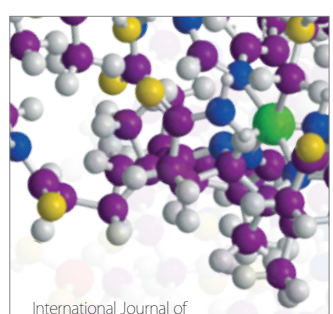

Carbohydrate Chemistry

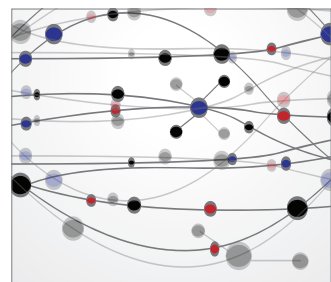

The Scientific World Journal
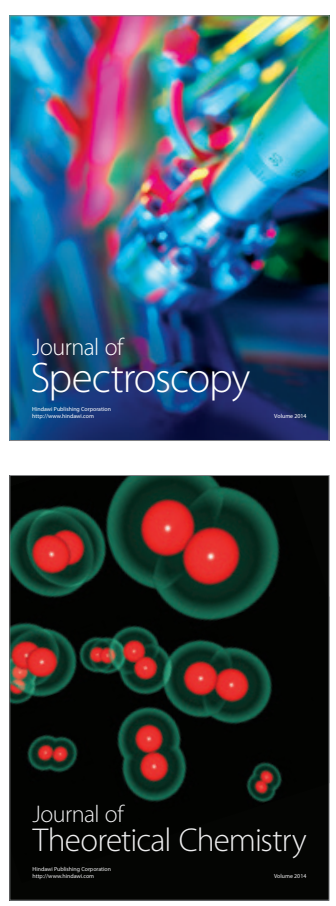
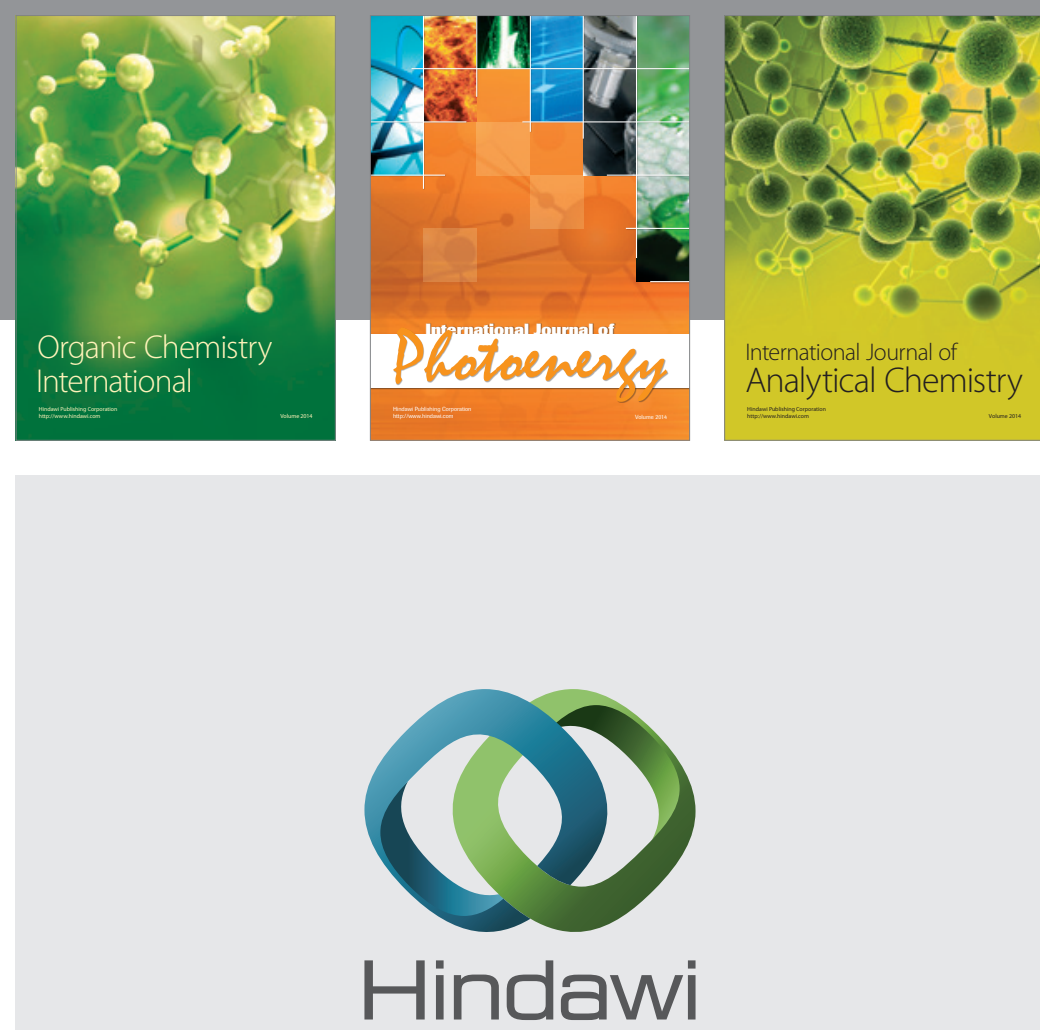

Submit your manuscripts at

http://www.hindawi.com
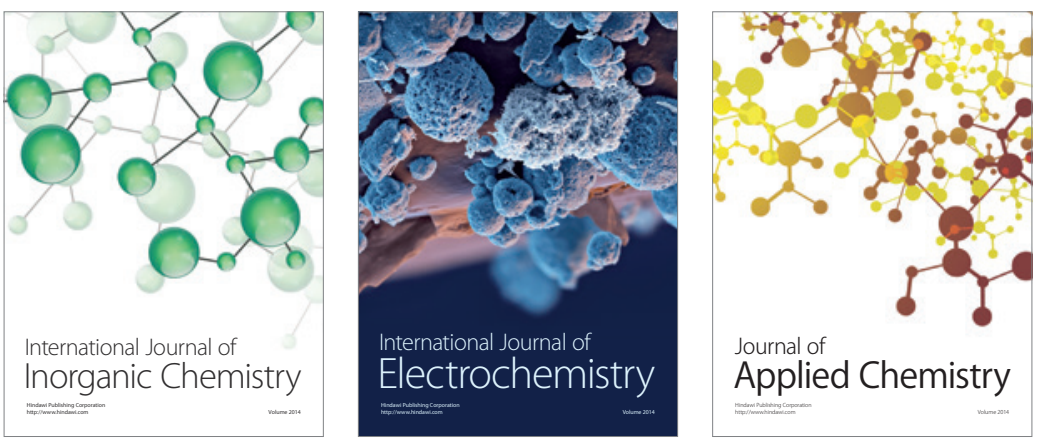

Journal of

Applied Chemistry
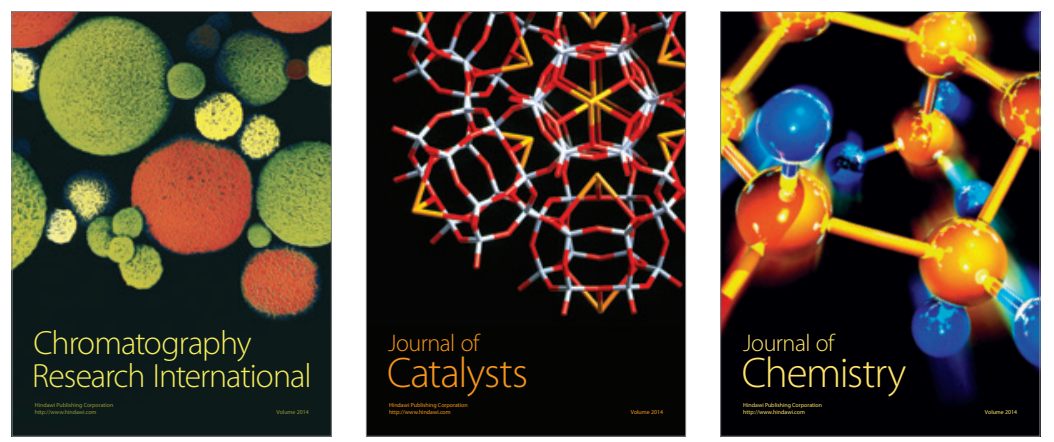
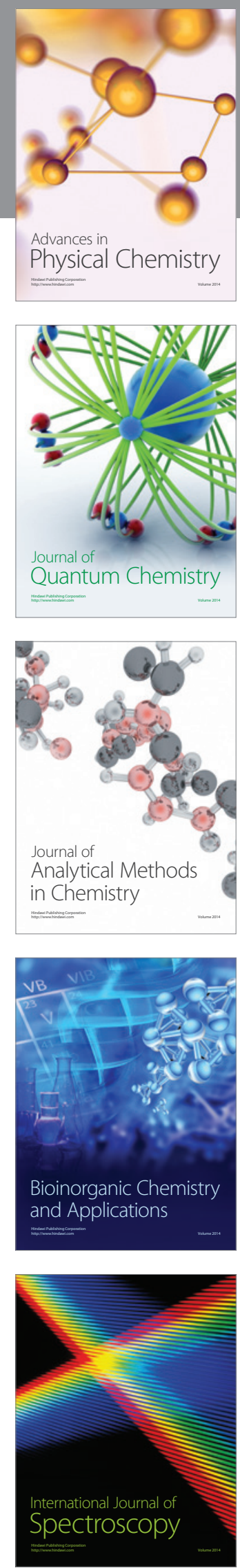